\title{
Searching for intergalactic star forming regions in Stephan's Quintet with SITELLE
}

\section{Ionised gas structures and kinematics ${ }^{\star}$}

\author{
S. Duarte Puertas ${ }^{1}$, J. Iglesias-Páramo ${ }^{1,2}$, J. M. Vilchez ${ }^{1}$, L. Drissen ${ }^{3,4}$, C. Kehrig ${ }^{1}$, and T. Martin ${ }^{3,4}$ \\ ${ }^{1}$ Instituto de Astrofísica de Andalucía - CSIC, Glorieta de la Astronomía s.n., 18008 Granada, Spain \\ e-mail: salvini@iaa.es \\ 2 Estación Experimental de Zonas Áridas - CSIC, Ctra. de Sacramento s.n., La Cañada, Almería, Spain \\ 3 Département de Physique, de Génie Physique et d'Optique, Université Laval, Québec, QC G1V 0A6, Canada \\ ${ }^{4}$ Centre de Recherche en Astrophysique du Québec, Québec, Canada
}

Received 12 April 2019 / Accepted 18 June 2019

\begin{abstract}
Stephan's Quintet (SQ), the prototypical compact group of galaxies in the local Universe, has been observed with the imaging Fourier transform spectrometer SITELLE, attached to the Canada-France-Hawaii-Telescope, to perform a deep search for intergalactic starforming emission. In this paper we present the extended ionised gaseous structures detected and analyse their kinematical properties. The large field of view $\left(11^{\prime} \times 11^{\prime}\right)$ and the spectral ranges of SITELLE have allowed a thorough study of the entire galaxy system, its interaction history and the main properties of the ionised gas. The observations have revealed complex three-dimensional strands in SQ seen for the first time, as well as the spatially resolved velocity field for a new SQ dwarf galaxy (M 82-like) and the detailed spectral map of NGC 7320c, confirming its AGN nature. A total of $175 \mathrm{SQ} \mathrm{H} \alpha$ emission regions have been found, 22 of which present line profiles with at least two kinematical components. We studied 12 zones and 28 sub-zones in the SQ system in order to define plausible physical spatial connections between its different parts in the light of the kinematical information gathered. In this respect we have found five velocity systems in SQ: (i) $v=[5600-5900] \mathrm{km} \mathrm{s}^{-1}$ associated with the new intruder and the southern debris region; (ii) $v=[5900-6100] \mathrm{km} \mathrm{s}^{-1}$, associated with the north starburst A and south starburst A and the strands connected to these zones; (iii) $v=[6100-6600] \mathrm{km} \mathrm{s}^{-1}$, associated with the strands from the large-scale shock region (LSSR); (iv) $v=[6600-6800] \mathrm{km} \mathrm{s}^{-1}$, associated with the young tidal tail, the starburst A (SQA), NGC 7319, and the NGC 7319 north lobe; and (v) $v=[6800-7000] \mathrm{km} \mathrm{s}^{-1}$, associated with the strands seen connecting LSSR with SQA. We fail to detect ionised gas emission in the old tail, neither in the vicinity of NGC 7318A nor in NGC 7317, and the connection between NGC 7319 north lobe and SQA cannot be confirmed. Conversely, a clear gaseous bridge has been confirmed both spatially and kinematically between the LSSR zone and the NGC 7319 AGN nucleus. Finally, a larger scale, outer rim winding the NGC 7318B/A system clockwise north-west to south-east has been highlighted in continuum and in $\mathrm{H} \alpha$. This structure may be reminiscent of a sequence of a previously proposed scenario for SQ a sequence of individual interactions.
\end{abstract}

Key words. galaxies: evolution - galaxies: groups: general - galaxies: kinematics and dynamics - galaxies: star formation

\section{Introduction}

Stephan's Quintet (SQ, Stephan 1877) is one of the most studied galaxy aggregates and it represents a unique laboratory for understanding how a compact group evolves. SQ is composed of elliptical (NGC 7317 and NGC 7318A) and spiral galaxies (NGC 7319, NGC 7320c, and NGC 7318B). NGC 7318B is a gas-rich galaxy that has experienced several interactions throughout its evolution (e.g. Moles et al. 1997; Sulentic et al. 2001). One foreground spiral galaxy (NGC 7320) with a discordant redshift (Burbidge \& Burbidge 1961) can also be seen in the field of view (FoV) of SQ.

Moles et al. (1997) and Sulentic et al. (2001) suggested that compact groups of galaxies are formed by acquiring intruder galaxies associated with a large-scale structure. All the galaxies of SQ, except NGC 7318B, have evolved dynamically, los-

\footnotetext{
* The data of the radial velocity and the $\mathrm{H} \alpha$ flux fitting are available at the CDS via anonymous ftp to cdsarc.u-strasbg. fr (130.79.128.5) or via http://cdsarc.u-strasbg.fr/viz-bin/ cat/J/A+A/629/A102
}

ing their interstellar medium (ISM). Moles et al. (1997) proposed an evolutionary model for SQ based on two intruders, an old intruder (OI, NGC 7320c) and a new intruder (NI, NGC 7318B). SQ is at an advanced state of interaction, evidenced by the existence of, for example, at least young and old tidal tails with ages of $150-200 \mathrm{Myr}$ and $400-500 \mathrm{Myr}$, respectively (Fedotov et al. 2011); a large-scale shock region (LSSR, e.g. Allen \& Hartsuiker 1972; Ohyama et al. 1998; IglesiasPáramo et al. 2012) produced by an ongoing interaction between NGC 7318B and both NGC 7319 and debris material from previous interactions; a diffuse gas halo close to NGC 7317 composed by old stars that indicates that the formation of SQ took place several Gyr ago (Duc et al. 2018).

From the kinematical point of view, SQ covers a large radial velocity range $\left(\sim 5500\right.$ to $\left.\sim 6600 \mathrm{~km} \mathrm{~s}^{-1}\right)$. The core of SQ (i.e. NGC 7317, NGC 7318A, and NGC 7319) and NGC 7320c show a mild radial velocity dispersion, whereas NGC 7318B has an unusually high radial velocity dispersion compared to the others $\left(\Delta V \sim 1000 \mathrm{~km} \mathrm{~s}^{-1}\right)$, indicating that it is not bound to the SQ (Trinchieri et al. 2005). Classically, two radial velocity structures 
were detected by Sulentic et al. (2001): the gas associated with the SQ and the gas associated with NI, where most of the emission detected in the ISM comes from the NI. Iglesias-Páramo et al. (2012) observed three radial velocity components but could only focus on the lower part of the shock. The LSSR spans a wide range of redshift (e.g. Konstantopoulos et al. 2014). Its structure and gas distribution have not been entirely examined until now due to the inability to fully study the system (from NGC 7320c to NGC 7317).

SQ has been widely researched with different wavelengths by many authors: with radio (e.g. Williams et al. 2002; Lisenfeld et al. 2004; Guillard et al. 2012); ultraviolet (e.g. Xu et al. 2005; de Mello et al. 2012); X-ray (e.g. Trinchieri et al. 2003; Heida et al. 2012); infrared (e.g. Appleton et al. 2006, 2017; Cluver et al. 2010; Guillard et al. 2009); and visible (e.g. Moles et al. 1998; Iglesias-Páramo \& Vílchez 2001; Sulentic et al. 2001; Mendes de Oliveira et al. 2004; Iglesias-Páramo et al. 2012; Trancho et al. 2012; Konstantopoulos et al. 2014; Rodríguez-Baras et al. 2014). There are also photometric studies (e.g. Gallagher et al. 2001; Fedotov et al. 2011; Duc et al. 2018) and simulations (e.g. Renaud et al. 2010; Hwang et al. 2012). Finally, several previous interferometric Fabry-Perot studies of the SQ (e.g. Sulentic et al. 2001; Mendes de Oliveira et al. 2001) have been done with diverse spatial coverages in wavelength-velocity resolution, though sometimes making difficult the discrimination between the emission of nearby lines at different velocities.

This work is based on the spectroscopic data from SITELLE (Grandmont et al. 2012), an imaging Fourier transform spectrometer (IFTS), attached to the Canada-France-Hawaii Telescope (CFHT). SITELLE allows us to study, for the first time, a large FoV considering the optical spectrum ranging from the UV atmosphere limit to the red, in order to study the physical, chemical, and kinematical properties of the entire SQ area. Our study presents an extensive spectral mapping that covers from the inner SQ "core" to the large gas halo surrounding the SQ. SITELLE observations offer us a great opportunity to perform an unbiased search for the star forming and tidal emission regions (e.g. tidal dwarf galaxies, TDG) present in the SQ in order to study their origin and properties. These data also provide a detailed spectroscopic mapping of the extended shock zone and its associated emission, with a seeing limited spatial resolution $\left(\sim 0.8^{\prime \prime}\right)$. This is relevant to obtain a more complete view of the ionisation structure of the shock and its environment, discriminating in velocity space the shock ionised gas from other intervening gaseous material (e.g. HII regions from the arms of the central spiral galaxies, gas associated with the AGN main galaxy).

The structure of this paper is organised as follows: in Sect. 2 we describe the data and the methodology used to select the $\mathrm{H} \alpha$ emission regions from the SQ. We detail our main results in Sect. 3. Finally, the discussion and main conclusions of our work are presented in Sect. 4. Line fluxes within the SN1, SN2, and SN3 data cubes, and the study of the physical properties and excitation conditions of the gas, will be presented in a future paper (Duarte Puertas et al., in prep., Paper II). Throughout the paper, we assume a Friedman-Robertson-Walker cosmology with $\Omega_{\Lambda 0}=0.7, \Omega_{\mathrm{m} 0}=0.3$, and $H_{0}=70 \mathrm{~km} \mathrm{~s}^{-1} \mathrm{Mpc}^{-1}$.

\section{Observations and data analysis}

\subsection{Observations}

SITELLE is an IFTS that aims to study the spatially-resolved spectra of extended sources. It has a FoV of $11^{\prime} \times 11^{\prime}\left(0.32^{\prime \prime}\right.$ per pixel, Drissen et al. 2019), suitable for investigating complex
Table 1. Observing parameters.

\begin{tabular}{cccc}
\hline \hline$(1)$ & $(2)$ & $(3)$ & $(4)$ \\
Filter & SN1 & SN2 & SN3 \\
\hline RA $($ h m s) & $22: 36: 05.30$ & $22: 36: 05.30$ & $22: 36: 05.84$ \\
Dec $\left({ }^{\circ} \prime \prime \prime\right)$ & $33: 58: 59.9$ & $33: 58: 59.9$ & $33: 59: 09.6$ \\
Observing date & $2016-07-09$ & $2016-07-10$ & $2015-08-07$ \\
& & & $2015-08-08$ \\
Spectral range $T>90 \%(\mathrm{~nm})$ & $363-386$ & $482-513$ & $648-685$ \\
Number of steps & 85 & 169 & 342 \\
Exp. time/step (s) & 75 & 65 & 25 \\
Total exp. time (h) & 1.77 & 3.05 & 2.37 \\
Mean resolution, R & 500 & 760 & 1560 \\
\hline
\end{tabular}

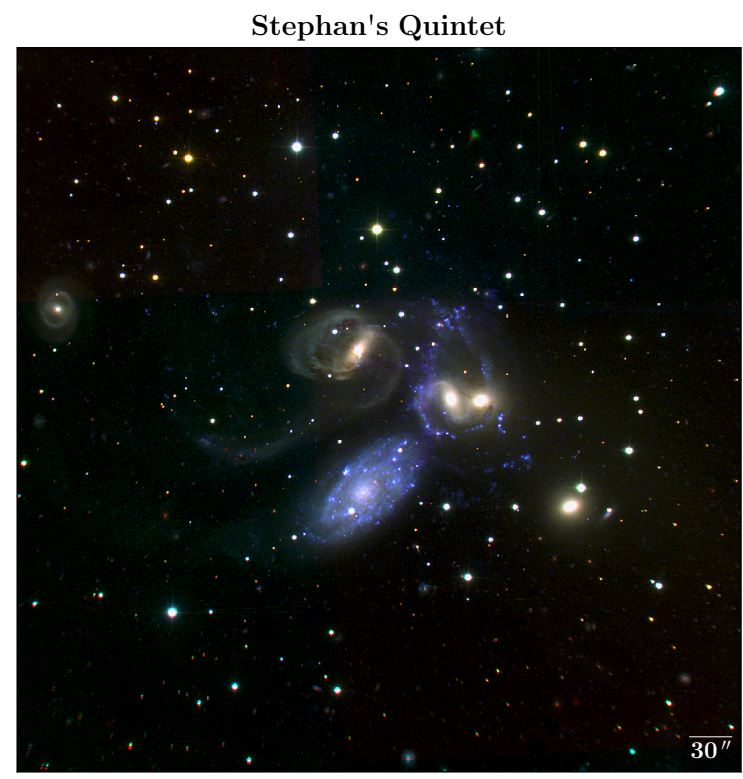

Fig. 1. SITELLE deep-colour image of SQ composed using SN1, SN2, and SN3 data cubes. North is top and east is left. The distance considered for SQ in this paper is $d=88.6 \mathrm{Mpc}$ (from the NASA/IPAC Extragalactic Database known as NED). At the distance of SQ, 30" corresponds to $\sim 13.04 \mathrm{kpc}$.

and extensive systems of galaxies such as SQ. The observations were carried out in August 2015 and July 2016 (P.I. Drissen) under an average seeing of $\sim 0.8^{\prime \prime}$. The three filters SN1, SN2, and SN3 were used to capture the emission lines of our interest: [O II] $\lambda 3727,[\mathrm{O}$ III] $\lambda \lambda 4959,5007, \mathrm{H} \beta,[\mathrm{N} \mathrm{II}] \lambda \lambda 6548,6583$, [S II] $\lambda \lambda 6716,6731$, and $\mathrm{H} \alpha$. Table 1 shows the observing parameters (right ascension or RA, declination or Dec, observing date, spectral range, number of steps, mean resolution, and the total exposure time) considered for each filter. Figure 1 shows a SITELLE deep-colour image of the SQ field composed using the integrated emission on the SN1, SN2, and SN3 data cubes (see Drissen et al. 2019, Sect. 5.4). We worked the data cubes in wavenumbers $\left(\mathrm{cm}^{-1}\right)$, which is SITELLE's natural units.

\subsection{Reduction, calibration, and measurements}

The data were fitted using the Python-based software ORCS (Martin et al. 2015). Data were recalibrated in wavelength using the $\mathrm{OH}$ sky lines that are visible in most parts of the FoV. We fitted the OH sky lines using the function SPECTRALCUBE.MAP_SKY_ VELOCITY and the resulting corrections were implemented to 
the cube using SpectralCube.correct_WAVELENGTH (Martin et al. 2018). For each data cube, we subtracted the sky using the median sky spectrum from three dark regions (2700 pixels were used). For illustrative purposes, we show the median sky spectrum in the SN3 data cube in Fig. 2. In order to obtain a more precise radial velocity correction we used the function GET_RADIAL_VELOCITY_CORRECTION. Through this function, we found a shift of $15 \mathrm{~km} \mathrm{~s}^{-1}$ that was added to the measured velocity. The flux calibration was performed using the data cube of the standard star GD71. The Galactic extinction is very small, $E(B-V)=0.07$, according to the NASA/IPAC Extragalactic Database known as $\mathrm{NED}^{1}$, and we did not correct for it. The methodology used to analyse the data involved several steps that we describe below.

\subsubsection{Searching for $\mathrm{H} \alpha$ emission}

We considered a binning $3 \times 3$ to amplify the signal-to-noise ratio ( $\sim 50000$ pixels). In addition a study was done only in $\mathrm{H} \alpha$ for a binning $6 \times 6$ (see Sect. 3.3 $)^{2}$ in order to unveil the lowest surface brightness gaseous emission in SQ. Subsequently, we searched for the wavenumber position $\left(k_{\max }\right)$ where the maximum intensity peak of the $\mathrm{H} \alpha$ emission line $\left(A_{\max }\right)$ is found in the typical wavenumber range of SQ $\left(\Delta K_{\mathrm{SQ}}=[14820,15021] \mathrm{cm}^{-1}\right)$ and NGC $7320\left(\Delta K_{\mathrm{NGC} 7320}=[15147,15239] \mathrm{cm}^{-1}\right)$, selecting the largest $A_{\max }$ between them. Once we found the position of the maximum intensity, we derived the standard deviation when the transmission curve is higher than $90 \%$ for the SN3 filter $\left(\operatorname{std}_{90}\right)$. To compute $\operatorname{std}_{90}$, the typical wavenumber range for the emission lines $\mathrm{H} \alpha$, [N II] $\lambda \lambda 6548,6583$, and [S II] $\lambda \lambda 6716,6731$ of each pixel have not been considered. We selected those pixels with $\frac{A_{\max }}{\text { std } 90} \geq 2.5$ ( $\sim 25800$ pixels $)$. We assumed that the maximum value corresponds to the $\mathrm{H} \alpha$ position. We know that the maximum in the AGN galaxy nucleus corresponds to [N II] $\lambda 6583$ and this effect will be corrected later. We used the $k_{\max }$ value to derive the possible initial velocity for $\mathrm{H} \alpha\left(v_{\text {ini }}(\mathrm{H} \alpha)\right)$ according to the following equation: $v_{\text {ini }}(\mathrm{H} \alpha)=\left[\left(\left(\frac{1 e 7}{k_{\max } \cdot \lambda(\mathrm{H} \alpha) \cdot 0.1}\right)-1\right) \cdot c\right]$, where $\lambda(\mathrm{H} \alpha)=6562.8 \mathrm{~nm}$ and $c=299792 \mathrm{~km} \mathrm{~s}^{-1}$.

\subsubsection{Identifying $\mathrm{H} \alpha$ emission regions}

We fitted the spectrum for every selected pixel using the $v_{\text {ini }}(\mathrm{H} \alpha)$ derived above. Initially, we fitted each spectrum to a sincgauss function (the convolution of a Gaussian with a sinc function) for $\mathrm{H} \alpha$ and $[\mathrm{N} \mathrm{II}] \lambda \lambda 6548,6583$ simultaneously. The theoretical relation [N II] $\lambda 6548 / 6583=0.333$ is considered. When the broadening of the $\mathrm{H} \alpha$ line was lower than $70 \mathrm{~km} \mathrm{~s}^{-1}$, we adjusted the spectrum using a sinc function (the instrumental line shape, Martin et al. 2016). When no emission was detected in [N II] 16583, we refitted the spectrum only for the $\mathrm{H} \alpha$ emission line. The output parameters are the radial velocity, broadening, intensity peak, flux, and the corresponding uncertainties, as well as the standard deviation of each spectrum. After fitting the $\mathrm{H} \alpha$ line, we selected the pixels with contrast $(\mathrm{H} \alpha)=\frac{A_{\text {fit }}}{\text { std }_{0}} \geq 5$, where $A_{\text {fit }}$ is the intensity peak of the $\mathrm{H} \alpha$ emission line obtained from the fitting, and $v_{\text {ini }}(\mathrm{H} \alpha) \geq 5000 \mathrm{~km} \mathrm{~s}^{-1}$ ( 950 pixels). Figure 3 shows the $\mathrm{H} \alpha$ flux (upper panel) and $\mathrm{H} \alpha$ radial velocity (lower panel) maps for the $\sim 2100$ pixels (binning $3 \times 3$ ) with contrast

\footnotetext{
1 http://ned.ipac. caltech.edu/

2 Binning $6 \times 6$ was chosen after an iterative process to maximise the the signal-to-noise ratio while minimising the contribution of the background sky.
}

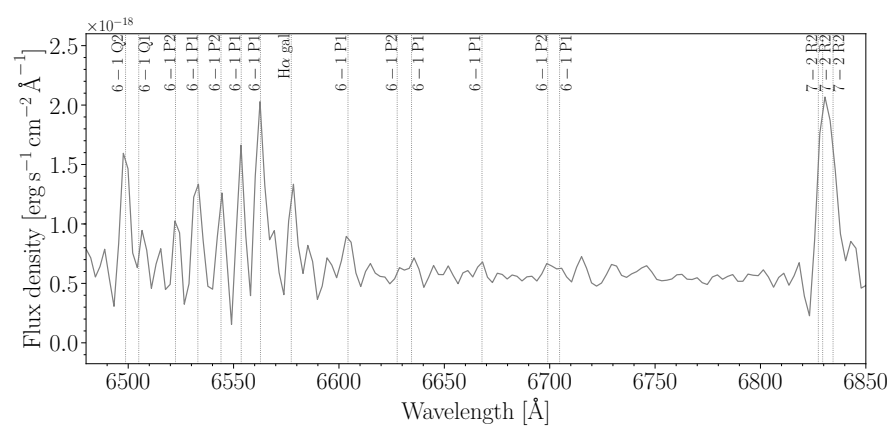

Fig. 2. Median sky spectrum in SN3 data cube (2700 pixels have been combined). The dashed vertical lines show multiple known $\mathrm{OH}$ sky lines.

$(\mathrm{H} \alpha) \geq 5$ and without velocity restrictions. We detect $\mathrm{H} \alpha$ emission in several galaxies: NGC 7319, NGC 7320c, NGC 7320 (the foreground galaxy with an average radial velocity of $786 \mathrm{~km} \mathrm{~s}^{-1}$, see inset plot in the lower panel for more details), and the new dwarf galaxy in SQ (NG, see Sect.3.4 and ID. 176 in Table B.1). Also, we detect $\mathrm{H} \alpha$ emission from the starbursts A and B (SQA and SQB, e.g. Xu et al. 1999). Additionally, this figure shows emission from the young tidal tail (YTT, e.g. Lisenfeld et al. 2002), LSSR, and the NI (e.g. Moles et al. 1997). We also detect emission to the left of NGC 7317; this region is discussed in depth in Sect. 3.4. From the sample of pixels selected above, we searched for all the $\mathrm{H} \alpha$ emission region candidates associated with the SQ. To do so, we first grouped the spatially associated emitting pixels. Then, the individual $\mathrm{H} \alpha$ regions associated with each group of pixels are defined searching for the local emission peaks in the $\mathrm{H} \alpha$ map using the Python-package ASTRODENDRO $^{3}$; The membership of each group of pixels to a given individual $\mathrm{H} \alpha$ region has been defined from the segmentation method using the Python packages SCIPY.NDIMAGE and SKIMAGE.MORPHOLOGY.WATERSHED. This method allows us to define a population of 209 SQ $\mathrm{H} \alpha$ region candidates.

\subsubsection{One velocity component analysis for each $\mathrm{SQ}$ $\mathrm{H} \alpha$ emission region}

For each $\mathrm{H} \alpha$ emission region we obtained the integrated spectrum adding the flux of its pixels; then all integrated spectra were fitted using the ORCS function ORCS.FIT_LINES _IN_INTEGRATED_REGION. The spectral lines $\mathrm{H} \alpha$ and [N II] $\bar{\lambda} \lambda \overline{65} 48,6583$ are fitted to a sincgauss function simultaneously, and when broadening of the $\mathrm{H} \alpha$ line is smaller than $70 \mathrm{~km} \mathrm{~s}^{-1}$, a sinc function was used, as detailed above. As the beginning of the SN3 transmission curve coincides with the emission lines [S II] $\lambda \lambda 6716,6731$ at SQ's redshift, we only take into account in the fit the spectral line [S II] $\lambda 6716$ for those $\mathrm{H} \alpha$ emission regions with radial velocities lower than $6260 \mathrm{~km} \mathrm{~s}^{-1}$, and [S II] $\lambda 6731$ for those $\mathrm{H} \alpha$ emission regions with velocities lower than $5760 \mathrm{~km} \mathrm{~s}^{-1}$. The output parameters are the radial velocity, broadening, intensity peak, total flux, and the corresponding uncertainties, as well as the standard deviation of each spectrum. The emission lines from the SN1 and SN2 data cubes were fitted using the velocity found in the previous step for $\mathrm{H} \alpha$ as a reference. It is important to note that we assumed the $\mathrm{H} \alpha$ radial velocity as the representative velocity for every $\mathrm{H} \alpha$

\footnotetext{
3 This tool estimates the background in our images and we searched the regions that have FWHM $\sim 9$ pixels and have peaks $\sim 3 \sigma$ above the background, from our sample of selected pixels.
} 

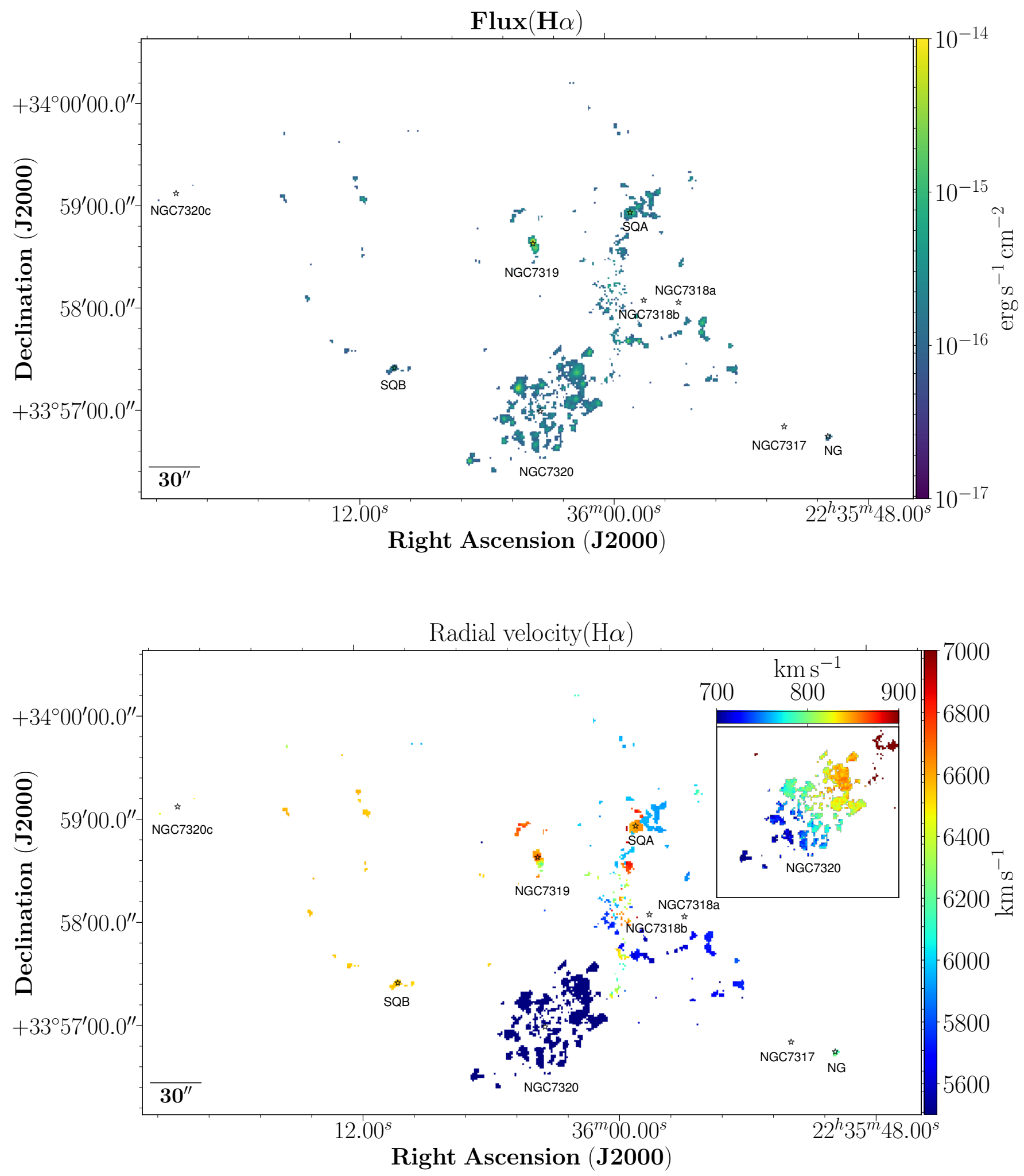

Fig. 3. Top panel: $\mathrm{H} \alpha$ flux map of SQ considering pixels with $\mathrm{H} \alpha$ contrast $\geq 5$. Bottom panel: $\mathrm{H} \alpha$ radial velocity map of SQ considering pixels with contrast $(\mathrm{H} \alpha) \geq 5$. The inset plot in the right panel indicates the radial velocity map of NGC 7320, the foreground galaxy, with contrast $(\mathrm{H} \alpha) \geq 5$ (see the text for details). NG (new dwarf galaxy) indicates the position of the new dwarf galaxy in SQ (see Sect. 3.4).

emission region. We followed the same methodology as previously described. For SN1 we fitted the spectral line [O II] 33727. For SN2 we fitted the lines $\mathrm{H} \beta$ and [O III] $\lambda \lambda 4959,5007$ in the wavenumber range $[19493,20746] \mathrm{cm}^{-1}$ (assuming the theoretical relation $[\mathrm{O} \mathrm{III}] \lambda 4959 / 5007=0.333)$. The standard deviation for the SN1 and SN2 filters ( $\left.\operatorname{std}_{90}\right)$ was derived as above. Figure 4 illustrates examples of the line fits for the SN1, SN2, and SN3 filters. All the line fluxes fitted in the SN1, SN2, and SN3 data cubes will be presented in a future paper (Duarte Puertas et al., in prep., Paper II). 


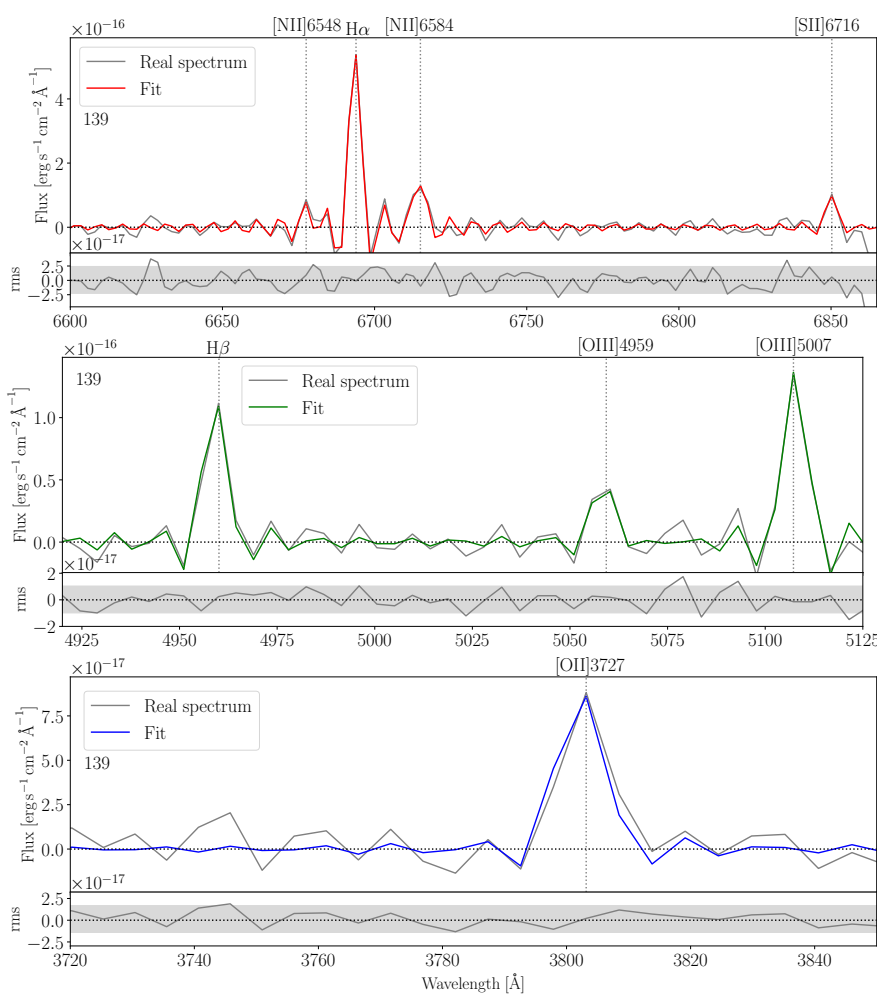

Fig. 4. Fitting example: spectra from region 139. In each upper panel, the grey line shows the real spectrum and the red, green, and blue coloured lines show the fit obtained with ORCS in the SN3, SN2, and SN1 filters, respectively. Upper panels: grey dotted lines show the location of the emission lines studied for this region. Lower panels: grey line shows the residual after the fit. The horizontal grey band indicates the $3 \sigma$ scatter.

\subsubsection{Complex kinematics spectral fitting of emission regions}

The one-velocity-component spectral fitting procedure produced unsatisfactory results for a selection of emission regions. A close visual inspection of these fits showed complex kinematics, with large velocity dispersion for the emission regions associated with the AGN galactic nucleus of NGC 7319 and the shock region (see Fig. 3 lower panel). Taking into account our spectral resolution and the signal-to-noise ratio, we studied only two velocity components for those emission regions with the velocity components resolved, as follows:

The H $\alpha,[\mathrm{N}$ II] $] \lambda \lambda 6548,6583$, and [S II] $\lambda \lambda 6716,6731$ emission lines of the spectrum of the SQ shock region were fitted simultaneously in the wavenumber range $[14820,15021] \mathrm{cm}^{-1}$ and with a sincgauss function. When one or both velocity components presented contrast in the $\mathrm{H} \alpha$ line lower than three, we assumed that they fit the criterion to be considered as one velocity components. Finally, for the rest of the emission lines ([O II $] \lambda 3727$, $\mathrm{H} \beta$, and [O III] $\lambda \lambda 4959,5007)$, we performed a two-component fit using the velocities and line widths derived from the $\mathrm{H} \alpha$ fit as input. We found $22 \mathrm{SQ} \mathrm{H} \alpha$ emission regions with line profiles that can be fit with two velocity components.

\subsection{Sample of $S Q H \alpha$ emission regions}

Following the methodology described above, we found 209 candidate emission regions in the SQ field. However, in this work we only focus on those regions that belong to SQ (hereafter, "SQ
$\mathrm{H} \alpha$ emission regions") according to the following criteria: (i) the radial velocity of the region is within the radial velocity range of SQ (between $\sim 5600$ and $\sim 7000 \mathrm{~km} \mathrm{~s}^{-1}$ ); and (ii) at least one additional emission line besides $\mathrm{H} \alpha$ has been detected in the data cubes. According to this criteria, $175 \mathrm{SQ} \mathrm{H} \alpha$ emission regions were found. From these regions, 22 present broad $\mathrm{H} \alpha$ profiles that have been fit using two velocity components. There may be more than two velocity components, but for our spectral resolution, the residuals of the fit produced are found to be within a band of width 3 sigma rms of the defined continuum window. We selected the minimum number of components obtained, considering the resolution and the signal-to-noise ratio of our data cubes.

In Figs. 5 and 6 we show the sample of 175 SQ H $\alpha$ emission regions found in this work. The location of the 15 extra $\mathrm{H} \alpha$ emitter regions presented in Table A.1. are also shown. In Table B. 1 we present the catalogue of these SQ $\mathrm{H} \alpha$ emission regions. In Col. 1 the region name is presented, Col. 2 shows the RA and Dec coordinates, Cols. 3 and 4 show the radial velocity and the area, Col. 5 indicates the corresponding subzone. We add the information for NGC 7320c which is discussed in Sect. 3.4 (ID 1 in Table B.1). We have compared our results with the recent works by Konstantopoulos et al. (2014) and IglesiasPáramo et al. (2012). From the $40 \mathrm{H} \alpha$ emitting regions presented in Konstantopoulos et al. (2014), 34 have the same $\alpha, \delta$ as our regions. Twenty-five of these regions can be directly compared since they present a single value of radial velocity. When compared with Iglesias-Páramo et al. (2012), which has lower spatial resolution, only six regions were identified (pointings $\mathrm{M}$ and $\mathrm{S}$ ). The mean of the differences between our $\mathrm{H} \alpha$ radial velocities and the ones in these previous works, $\Delta v$, is $\Delta v=1.1 \pm 14.9 \mathrm{~km} \mathrm{~s}^{-1}$, which is consistent to within the errors.

For the sake of the analysis of the sample, in the following we separate the SQ emission regions in two sub-samples: lower radial velocity sub-sample (LV) composed of those regions where the radial velocity $\leq 6160 \mathrm{~km} \mathrm{~s}^{-1}$ (for the two velocity components fit, the lowest radial velocity was considered); conversely, the higher radial velocity sub-sample (HV) is defined including all the regions with radial velocity $>6160 \mathrm{~km} \mathrm{~s}^{-1}$ (for the two velocity components fit, the greatest radial velocity was considered). The nominal radial velocity of $6160 \mathrm{~km} \mathrm{~s}^{-1}$ was chosen as a limit value to highlight the discrete emission features associated with NI (NGC 7318B).

Figure 7 presents the different zones and subzones defined by the $175 \mathrm{SQ} \mathrm{H} \alpha$ emission regions (see below). In Fig. 8 we show the radial velocity versus RA diagram in the upper right panel; Dec versus radial velocity diagram in the upper right panel; and a three-dimensional view (i.e. RA - Dec - radial velocity diagram) of SQ $\mathrm{H} \alpha$ emission regions in the lower panel. From the three-dimensional information obtained considering Figs. 7 and 8, we have defined 12 zones and 28 subzones in SQ. We have divided the zones (and thus the subzones) in the following way: (i) YTT (e.g. Lisenfeld et al. 2002), so that north and south strands are respectively YTTN and YTTS, and NGC 7320c is the old intruder (OI); (ii) NGC 7319 (NGC 7319 nucleus, NGC 7319 "arm", north lobe); (iii) $\mathrm{H} \alpha$ "bridge"; (iv) high radial velocity strands, Hs (H1 and H2); (v) SQA (e.g. Xu et al. 1999); (vi) low radial velocity strands, Ls (L1, L2, L3, and L4); vii) shock strands, Shs (Sh1, Sh2, Sh3, and Sh4); (viii) north and south of SQA (NSQA and SSQA, respectively); (ix) tidal tail at north of NSQA (NW, e.g. Renaud et al. 2010); (x) NI (e.g. Moles et al. 1997) strands, NIs (NI1, NI2, NI3, NI4, and NI5); (xi) southern debris region (SDR, e.g. Fedotov et al. 2011); and (xii) NG (see Sect. 3.4). Also, the 15 extra $\mathrm{H} \alpha$ emitter regions 


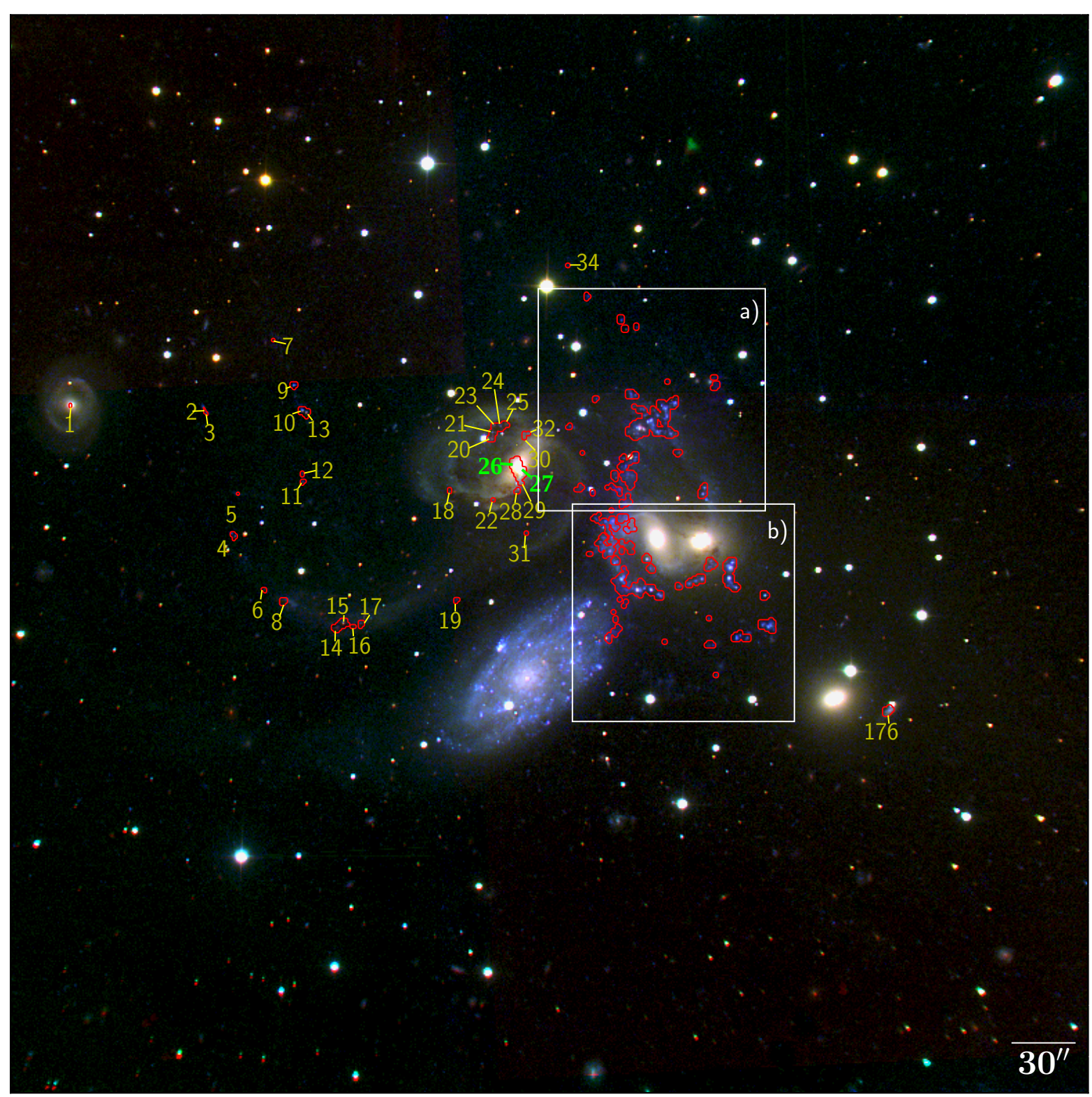

Fig. 5. SITELLE composite image from deep SN1, SN2 and SN3 data cubes of SQ. The red lines represent the H $\alpha$ contours corresponding to all $175 \mathrm{SQ} \mathrm{H} \alpha$ emission regions defined in Sect. 2.3. The H $\alpha$ regions from YTT, NGC 7319, NGC 7320c, and NG are labelled according to the nomenclature from Table B.1. Green and yellow labels identify the SQ H $\alpha$ regions showing a broad and narrow line profile, respectively. A zoomed-in view of the areas marked with the white rectangles are shown in Fig. 6.

from Appendix A are shown. Section 3.3 explains in more detail the connections between each zone and subzone. For each zone a detailed study was made to understand better the kinematic and chemical processes acting on SQ (see Sect. 3 and Duarte Puertas et al., in prep., Paper II).

\section{Results}

\subsection{Kinematical properties of $S Q H \alpha$ emission regions}

Figure 9 shows the radial velocity maps for the 175 SQ $\mathrm{H} \alpha$ regions. The SQ H $\alpha$ regions have been catalogued in two velocity sets, LV and HV, according to the definitions given in Sect. 2.2. Traditionally, two velocity ranges were observed in the shock zone. However, Iglesias-Páramo et al. (2012) showed an additional velocity range in the shock region. As can be seen in Fig. 3, at least five different velocity ranges are clearly distinguished, therefore, we can see two more velocity components. For low radial velocity components, two radial velocity ranges are shown: (i) $v=[5600-5900] \mathrm{km} \mathrm{s}^{-1}$, associated with the NIs and the SDR; (ii) $v=[5900-6100] \mathrm{km} \mathrm{s}^{-1}$, associated with the north of NSQA, SSQA, NW, and the Ls. For high radial velocity components, three radial velocity ranges are shown: (i) $v=[6100-6600] \mathrm{km} \mathrm{s}^{-1}$, associated with the Shs, connecting the part of SQ with high radial velocities with the lower radial velocities; (ii) $v=[6600-6800] \mathrm{km} \mathrm{s}^{-1}$, associated with the YTT, SQA, and AGN north lobe; (iii) $v=[6800-7000] \mathrm{km} \mathrm{s}^{-1}$, associated with the Hs, the strands that connect Shs with SQA, and those regions of strands Sh3 and Sh4 (e.g. ID 56, 80b, 85, 89b, and 113) that are closest to the Hs strands.

The upper panel of Fig. 9 shows a radial velocity gradient that was found in the SQ $\mathrm{H} \alpha$ emission regions from NI2 to SSQA, NI2 to NI3, and NI2 to NI5, increasing northwards up to $6100 \mathrm{~km} \mathrm{~s}^{-1}$ (the minimum radial velocity is in region 

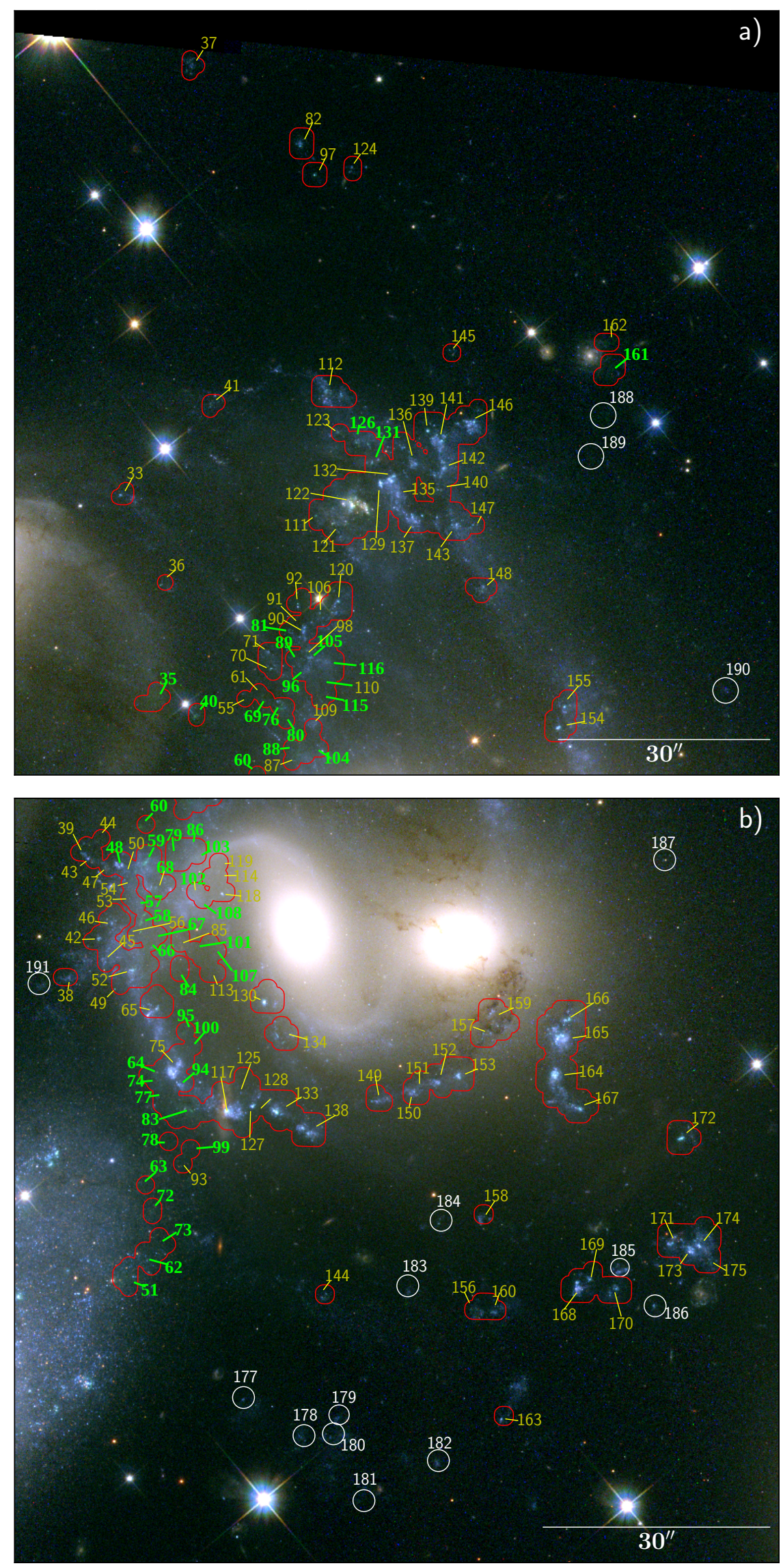

Fig. 6. Zoomed-in view of image from Hubble Space Telescope Wide Field Camera 3, also known as HST/WFC3 (PID 11502, PI Keith S. Noll) showing regions $a$ and $b$ from Fig. 5. The red circles and labels are defined as in Fig. 5. White circles show the location of the 15 extra H $\alpha$ emitter regions presented in Table A.1. Green and yellow labels identify the SQ H $\alpha$ regions showing a broad and narrow line profile, respectively. 


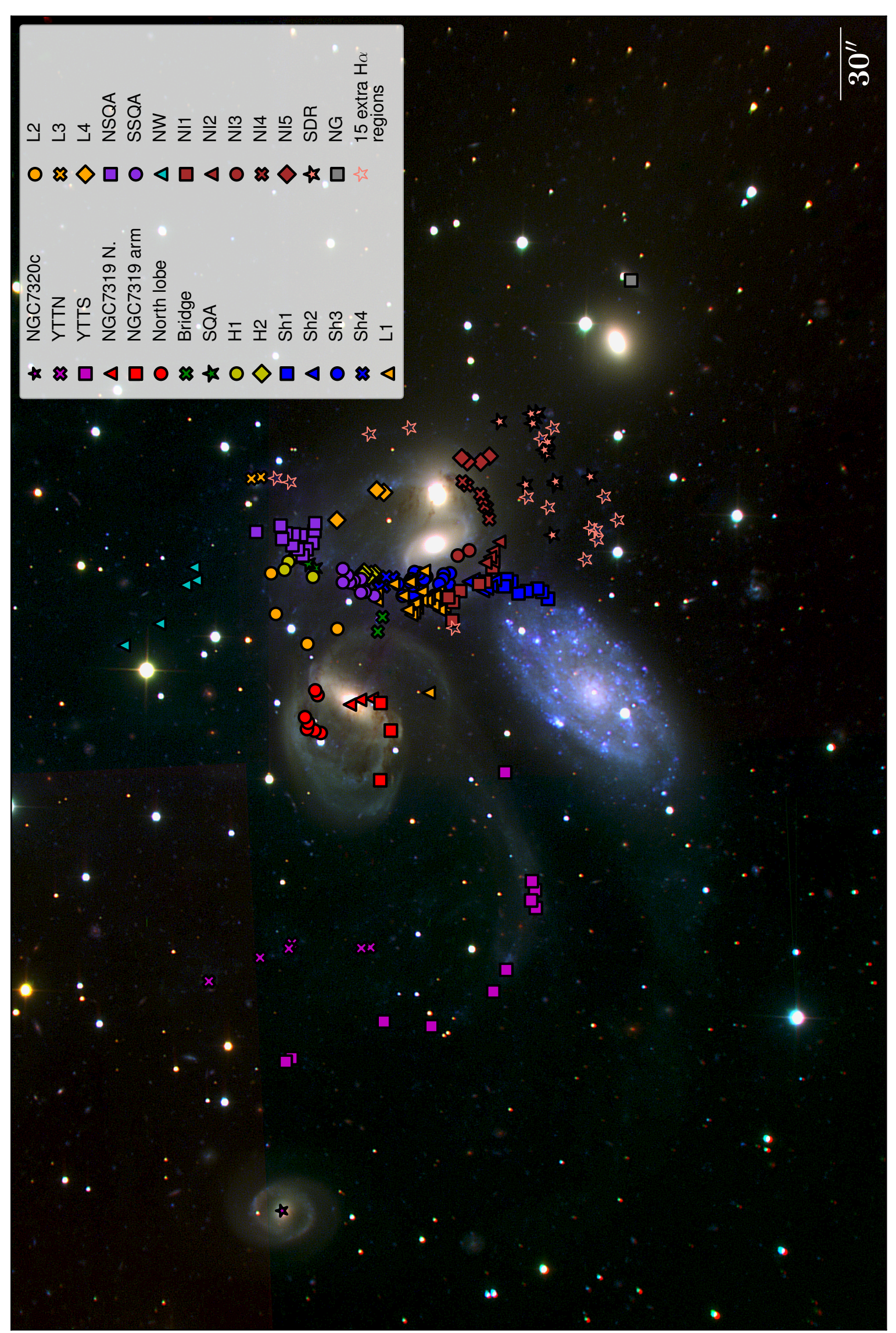

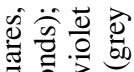

宓过 可

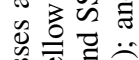
西 ज्ञ 和罂

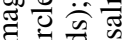
a.

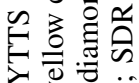

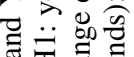
迅矿 Z保

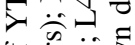
o 政

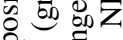
过语 on

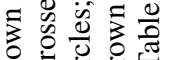
के 0 .

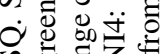
的荡江 to 80 : D. $\exists$ 泮 吾 을. 긍

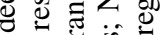
던 is 氠总要

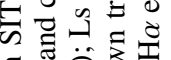
б चै

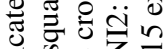
列 可 o. 过 0 잉 을 o 的艺 ते

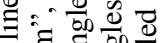
施

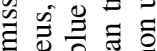
讨 岂 yo 230 क्ष

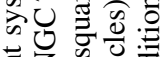
它乙

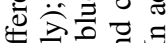
至 $\therefore$ 旸递

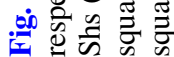



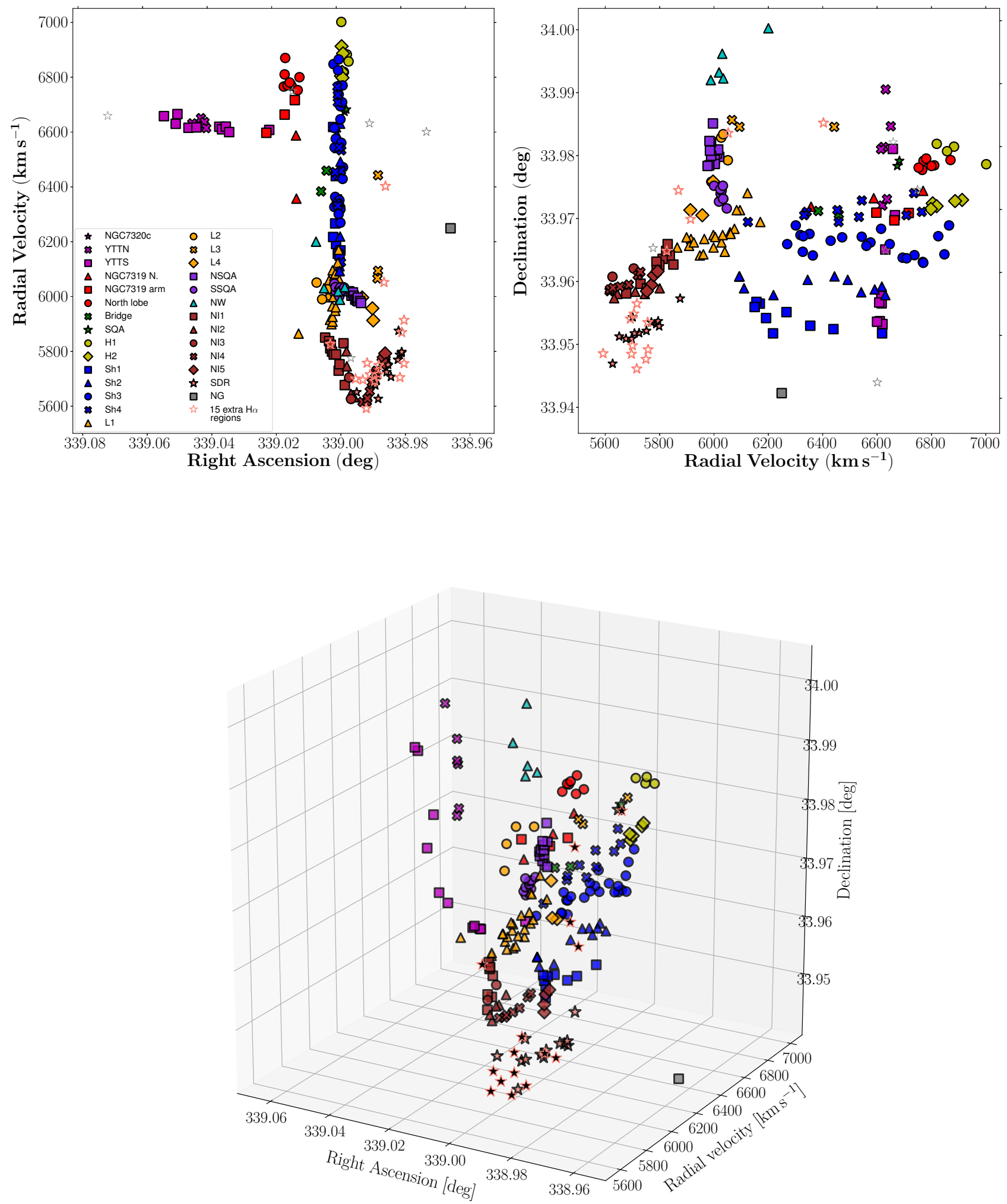

Fig. 8. Upper left panel: radial velocity versus RA diagram. Upper right panel: Dec versus radial velocity diagram. Lower panel: three-dimensional view of SQ $\mathrm{H} \alpha$ emission regions (RA, Dec, and radial velocity diagram). All the points in the figures have the same colours and markers as Fig. 7. In both upper panels, the nuclei of NGC 7320c, NGC 7319, NGC 7318B, NGC 7318A, and NGC 7317 are marked with an unfilled grey star.

149 , radial velocity $\sim 5610 \mathrm{~km} \mathrm{~s}^{-1}$ ). Located north of NSQA we can see the tidal tail NW with radial velocity $\sim 6000 \mathrm{~km} \mathrm{~s}^{-1}$. According to the simulations from Renaud et al. (2010), NW could have been formed by the merger between NGC 7318B and NGC 7318A. We have no clear evidence of this, but we believe that NSQA and SSQA have participated in this formation. This possible large-scale structure, which could be linked with the region $145\left(v=5994 \mathrm{~km} \mathrm{~s}^{-1}\right)$, is revealed. NI strands and SDR have lower radial velocities than $\sim 5900 \mathrm{~km} \mathrm{~s}^{-1}$. Moreover, regions with radial velocities higher than $6160 \mathrm{~km} \mathrm{~s}^{-1}$ are presented in 

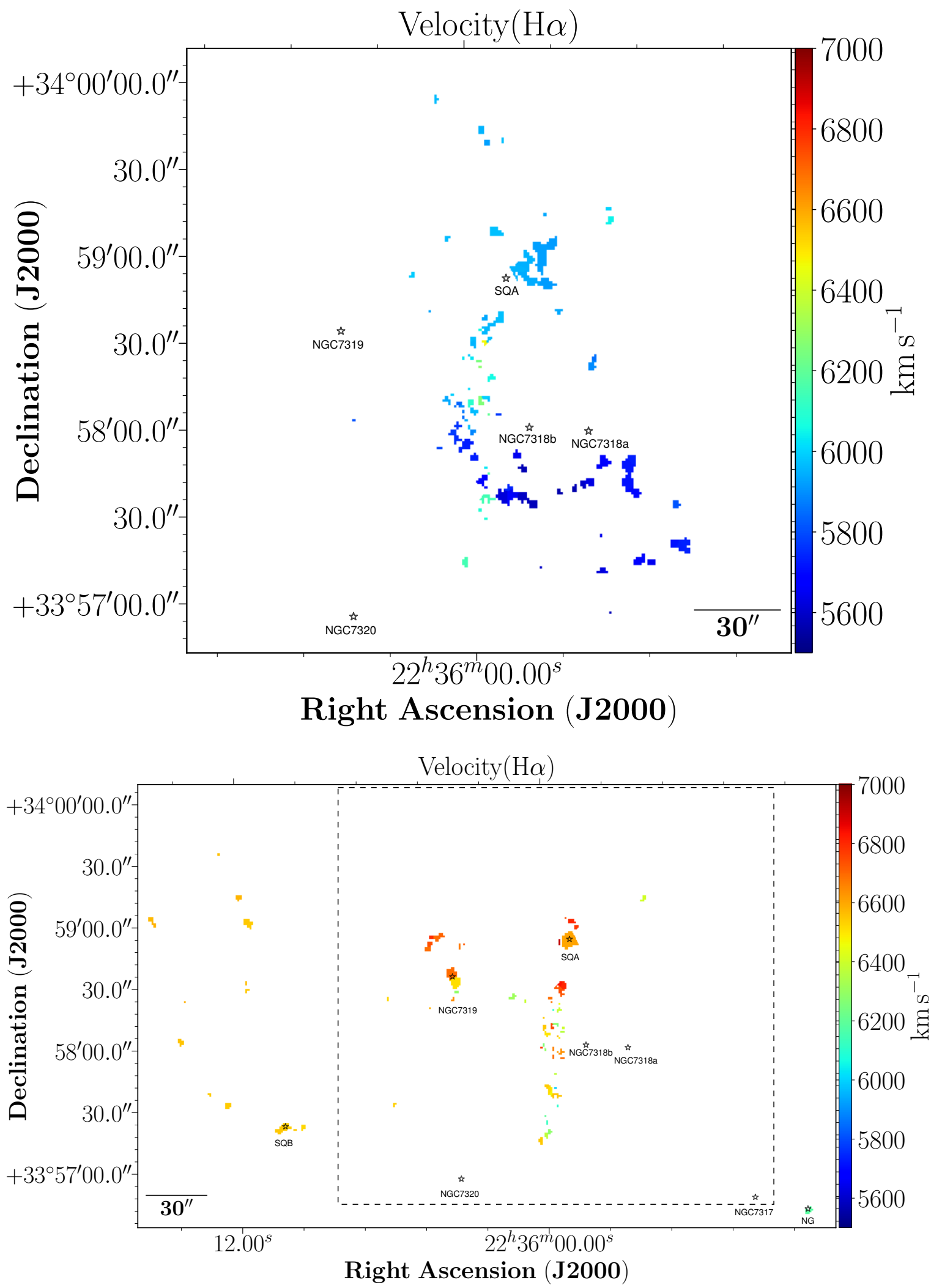

Fig. 9. SQ spatial map, colour-coded according to radial velocity of $\mathrm{H} \alpha$ for LV sample (upper panel) and HV sample (lower panel). The box in the lower panel indicates the zoomed zone shown in the upper panel.

Fig. 9 (lower panel). We detected several strands (Shs) that connect $\mathrm{HV}$ with $\mathrm{LV}$ parts covering a range of radial velocities from $\sim 6100$ to $\sim 6600 \mathrm{~km} \mathrm{~s}^{-1}$. SQA has radial velocity $\sim 6670 \mathrm{~km} \mathrm{~s}^{-1}$. Additionally, the north lobe has radial velocities from 6750 to
$6870 \mathrm{~km} \mathrm{~s}^{-1}$. We found two SQ regions in the $\mathrm{H} \alpha$ "bridge" (radial velocity $\sim 6400 \mathrm{~km} \mathrm{~s}^{-1}$, see Sect.3.3 for more information). The strands $\mathrm{H} 1$ and $\mathrm{H} 2$ connect Shs with SQA. The radial velocity and the Dec increase in H1 up to region 111 (radial velocity 


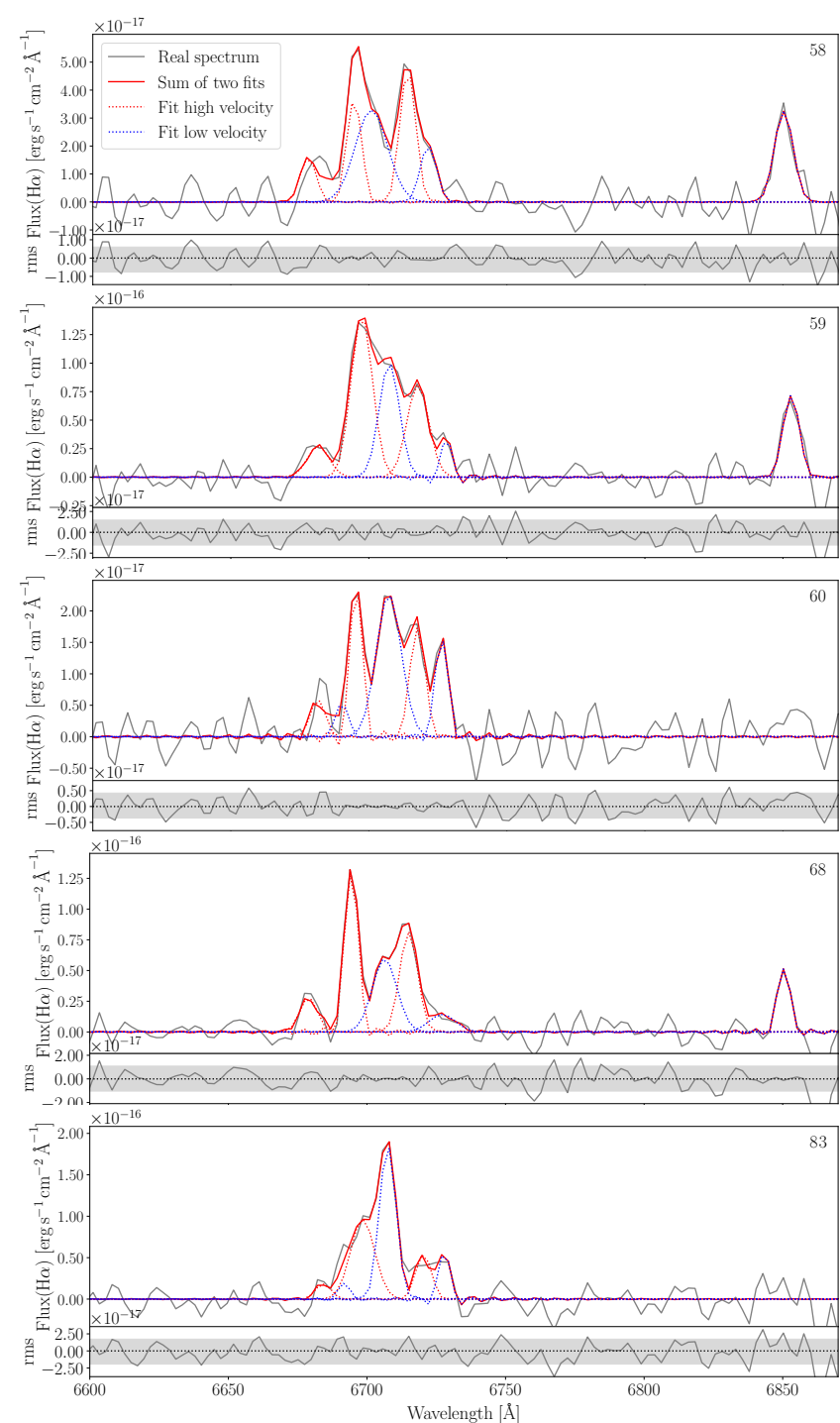

Fig. 10. From top to bottom: examples of the line fits from regions 58, $59,60,68$, and 83 with a broad line profile. In each upper panel, the grey line shows the real spectrum, and the red and blue dotted lines correspond to the fit of the low and high velocity components. The red solid line shows the sum of the two velocity component fits. The name of the region is indicated in each upper panel (top right). Lower panels: grey line shows the residual after the fit. The horizontal grey band indicates the $3 \sigma$ scatter.

$\sim 7000 \mathrm{~km} \mathrm{~s}^{-1}$ ). From region 111 to SQA (in H2) the radial velocity decreases. The NGC 7319 nucleus (regions 26, 27, and 29) shows radial velocities between $\sim 6350$ and $6760 \mathrm{~km} \mathrm{~s}^{-1}$ as a result of the outflow from the nucleus (Rodríguez-Baras et al. 2014). It is noteworthy that the NGC 7319 "arm" has regions with radial velocities from $\sim 6710$ (in region 28) to $\sim 6600 \mathrm{~km} \mathrm{~s}^{-1}$ (in region 18). We do not discard the possibility that it is produced by the interaction between NGC 7319 and another galaxy and it will remain as a conatus of tidal tail. The YTT has an average radial velocity of $\sim 6620 \mathrm{~km} \mathrm{~s}^{-1}$. YTT increases its radial velocity as we move away from NGC 7319. Another interesting finding is the NG (see Sect. 3.4).

\subsection{Regions with broad line profile}

As we can see in Sect. 2.3, SQ presents regions with broad and narrow line profiles. The regions showing a broad line profile are

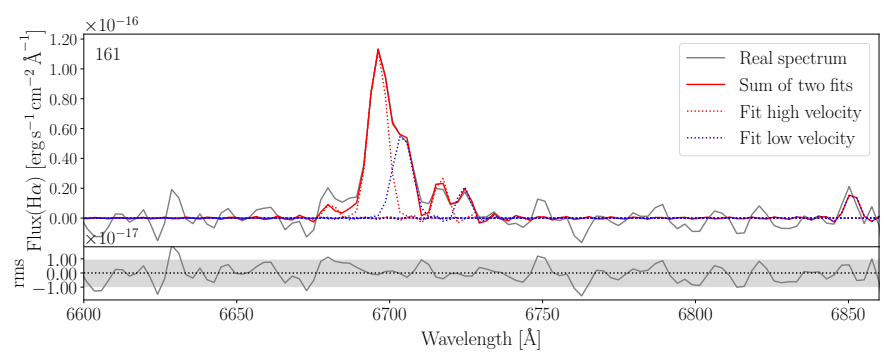

Fig. 11. Same as Fig. 10 but for region 161.
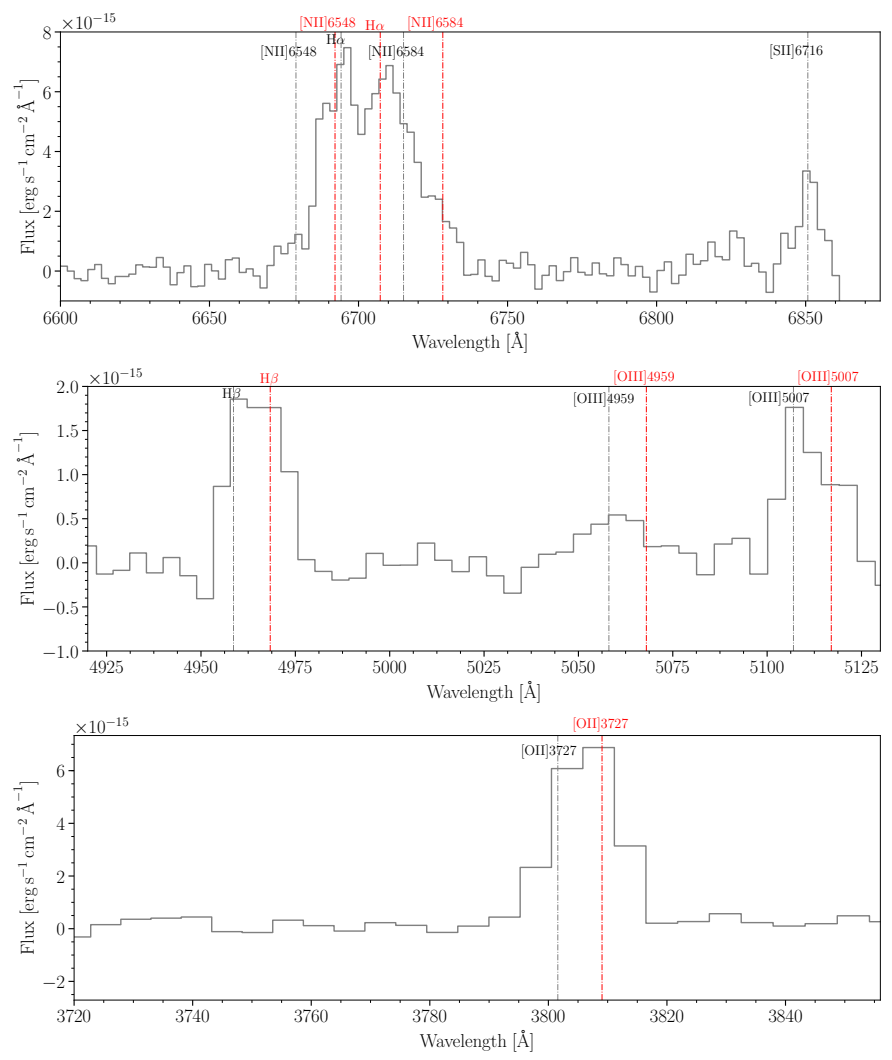

Fig. 12. Integrated spectra from large scale shock region in SN3, SN2, and SN1 data cubes. The black and red vertical dashed lines show the position of the emission lines studied at $\mathrm{H} \alpha$ radial velocities of $6000 \mathrm{~km} \mathrm{~s}^{-1}$ and $6600 \mathrm{~km} \mathrm{~s}^{-1}$, respectively.

located in the LSSR, the AGN nucleus of NGC 7319, and the L1 and L4 zones. In Fig. 10 we show several examples of these individual regions (noted as ID 58, 59, 60, 68, 83 in Table B.1) across the LSSR, and their corresponding fits using two sincgaussian functions, as explained in Sect. 2.3. A close visual inspection has also been carefully performed of all the fits of the broad line profile.

In Fig. 11 we present the broad $\mathrm{H} \alpha$ profile we found for region 161 located in the L4 area, a somewhat outlying zone (see Fig. 7), and its two velocity component fits. As far as we know, this broad profile is presented here for the first time for region 161. It is tempting to associate this broad profile region with a nearby secondary maximum apparent in the X-Ray maps of Trinchieri et al. (2005). However, the precise nature and the excitation of this gas still has not been ascertained.

In order to show the wide range of velocities encompassed within LSSR, its integrated emission is presented in Fig. 12 in the SN1, SN2, and SN3 filter windows. To obtain these 

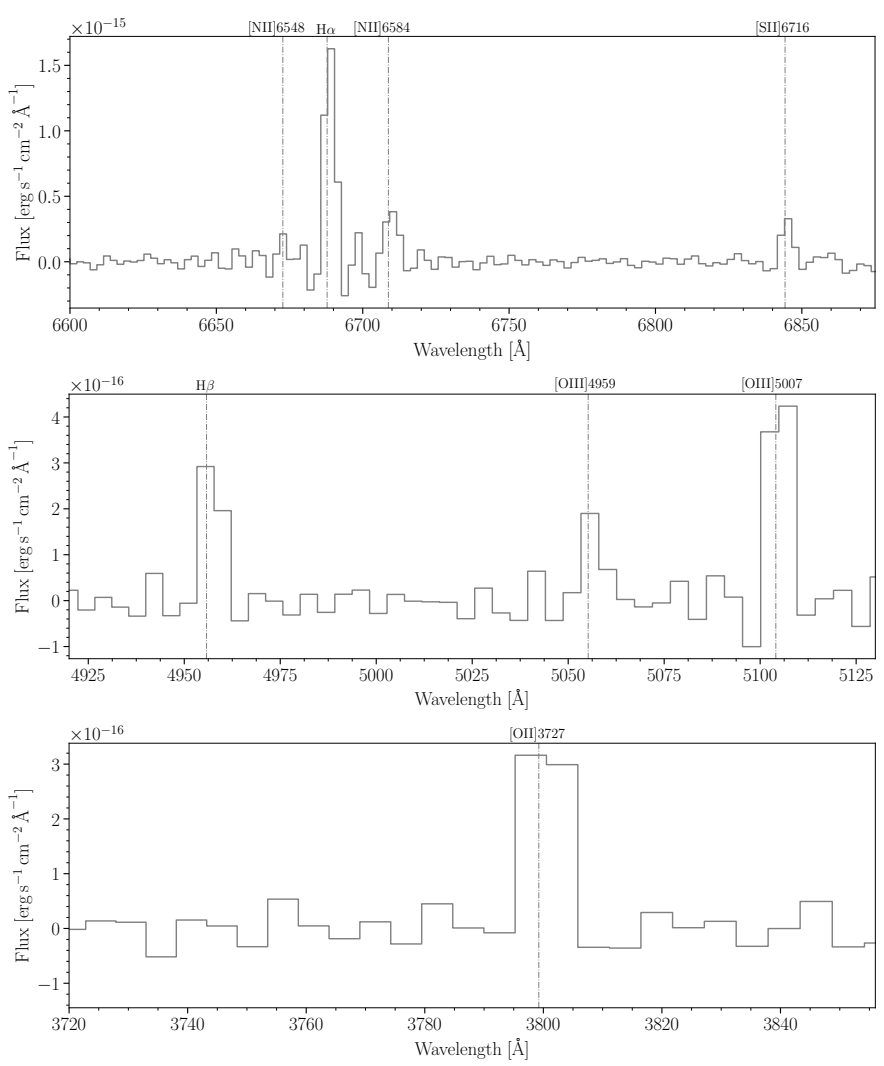

Fig. 13. Integrated spectra from $\mathrm{SQ} \mathrm{H} \alpha$ region 164 in $\mathrm{SN} 3, \mathrm{SN} 2$, and SN1 data cubes.

spectra, we considered all the $\mathrm{H} \alpha$ emission detected (binning $6 \times 6$ and contrast $\geq 2$ ) with $\mathrm{H} \alpha$ radial velocities $\geq 5000 \mathrm{~km} \mathrm{~s}^{-1}$ within a rectangle centred on the coordinate $\mathrm{RA}=339 \mathrm{deg}$ and Dec $=33.966 \mathrm{deg}$, with a width of $\Delta \mathrm{RA}=27.786 \mathrm{arcsec}$ and a height of $\Delta \mathrm{Dec}=99.512 \mathrm{arcsec}$ (defined according to the definition of the flux, as determined in the next section). As expected, the integrated spectra from LSSR show broad lines and several predominant peaks (e.g. $\mathrm{H} \alpha$ and [N II] $\lambda 6584$ ). For illustrative purposes, these can be compared with a typical narrow-lined $\mathrm{H} \alpha$ emission region (i.e. ID 164; Fig. 13).

\subsection{Unveiling low surface brightness $\mathrm{H} \alpha$ emission}

In order to detect the low surface brightness $\mathrm{H} \alpha$ emission regions, we produced a deep overview of all the $\mathrm{H} \alpha$ emission of the SQ system in Fig. 14. This was produced using a binning $6 \times 6$, contrast $(\mathrm{H} \alpha) \geq 2$, and radial velocities in the range of the SQ, in order to increase the detectability of the low surface brightness emission, as explained in Sect. 2.2. From a statistical analysis of our $\mathrm{H} \alpha$ flux measurements, we find that below a value of $\mathrm{H} \alpha$ flux per pixel of $6 \times 10^{-18} \mathrm{erg} \mathrm{s}^{-1} \mathrm{~cm}^{-2}$ we can distinguish a very low surface brightness $\mathrm{H} \alpha$ component besides the SQ $\mathrm{H} \alpha$ emission regions measured. The upper right panel of Fig. 14 shows a zoomed-in view of the LSSR, whereas the lower panels, as in Fig. 8, shows an overlapped view of the symbols corresponding to the very low surface brightness regions detected with the SQ H $\alpha$ emission regions found in Sect. 2.3. This low surface brightness gas emission represents different components spread throughout the whole system. This gives us important information about the connections between the different SQ zones (see Sect. 2.3) and helps us to better understand the complexity of SQ.
At lower radial velocities, $v \leq 6160 \mathrm{~km} \mathrm{~s}^{-1}$ (see lower panels in Fig. 14), we observe at least two subsystems that were probably in collision. On one hand, NI (brown symbols) ${ }^{4}$ colliding with the debris field in the shock path at L1 strand (orange triangles), on the other hand, the interaction between NSQA and SSQA (violet squares and circles, respectively). While the collision between NI and the debris field in the LSSR appears to be ongoing at this moment (e.g. Iglesias-Páramo et al. 2012), our observations indicate that the interaction between NSQA and SSQA has had at least one previous collision. Several strands of ionised gas emerging from NSQA are found here: to the north of NSQA a tidal tail, NW (cyan triangles) is indicated, while on the right we see the strand L3 (which increases towards higher radial velocities, $\sim 6470 \mathrm{~km} \mathrm{~s}^{-1}$, orange crosses) and the strand L4 (that goes southwards and could link with NI, orange diamonds). The strand L2 (orange circles) connects NSQA with SSQA, which reinforces the idea that NSQA and SSQA have had at least one previous interaction. The strand L1 joins the system formed by NSQA and SSQA with NI1. We must bear in mind that the majority of the regions from the strand L1 are compatible with fast shocks without precursor for solar metallicity and low density, with velocities between $175 \mathrm{~km} \mathrm{~s}^{-1}$ and $300 \mathrm{~km} \mathrm{~s}^{-1}$ from Allen et al. (2008) models (Duarte Puertas et al., in prep., Paper II).

At intermediate radial velocities (i.e. from $v \sim 6160$ to $\sim 6660 \mathrm{~km} \mathrm{~s}^{-1}$ ), several strands can be seen connecting Hs with Ls through the Shs strands (namely Sh1, Sh2, Sh3, and Sh4 represented by blue squares, triangles, circles, and crosses, respectively; see Figs. 8, upper right panel, and 14). It seems clear that this gas is a product of the interaction processes that occurred in SQ between NI and the debris field in the shock, although understanding these processes becomes complex. We also see at radial velocities $\sim 6360 \mathrm{~km} \mathrm{~s}^{-1} \mathrm{H} \alpha$ flux that connects the shock (Sh4 strand) with NGC 7319, into an $\mathrm{H} \alpha$ "bridge" (green crosses). This bridge has also been observed at other wavelength ranges (e.g. molecular hydrogen bridge detected: Cluver et al. 2010; Guillard et al. 2010; Appleton et al. 2013, 2017). In Fig. 14 (lower left panel) we can appreciate that this connection extends several hundred $\mathrm{km} \mathrm{s}^{-1}$.

At higher radial velocities, $v>6660 \mathrm{~km} \mathrm{~s}^{-1}$ we distinguish several gas strands. On one hand, from the NGC 7319 nucleus (red triangles) an outflow emerges, from radial velocities $\sim 6760 \mathrm{~km} \mathrm{~s}^{-1}$ to $\sim 6350 \mathrm{~km} \mathrm{~s}^{-1}$, in agreement with RodríguezBaras et al. (2014). On the other hand, the NGC 7319 "arm" (red squares) emerges up to radial velocities $\sim 6600 \mathrm{~km} \mathrm{~s}^{-1}$ westward towards YTT (magenta crosses and squares). The AGN north lobe zone (red circles) covers velocities higher and lower than that of the NGC 7319 nucleus $\left(\Delta v \sim 200 \mathrm{~km} \mathrm{~s}^{-1}\right)$. The right part of the north lobe seems to reach the NGC 7319 "arm". Conversely, in the shock part we see the strands $\mathrm{H} 1$ and $\mathrm{H} 2$ (yellow circles and triangles). These strands connect Shs with SQA (green stars) in the following ways: (i) from Shs (radial velocity $\sim 6660 \mathrm{~km} \mathrm{~s}^{-1}$ ) to the most distant region detected (region 111 , radial velocity $\sim 7000 \mathrm{~km} \mathrm{~s}^{-1}$ ) through the strand $\mathrm{H} 2$; and (ii) from region 111 to SQA (radial velocity $\sim 6670 \mathrm{~km} \mathrm{~s}^{-1}$ ) through strand $\mathrm{H} 1$.

We fail to detect ionised gas neither in the old tail, nor in the vicinity of NGC 7318A in agreement with Moles et al. (1998), nor in NGC 7317 in agreement with Duc et al. (2018). Neither do we find evidence that there is a gas connection between the NGC 7319 north lobe and SQA. Finally, we do not detect gas connecting the LSSR and NI5, nor in SDR (salmon stars).

\footnotetext{
4 All symbols referenced in this subsection come from Fig. 7.
} 

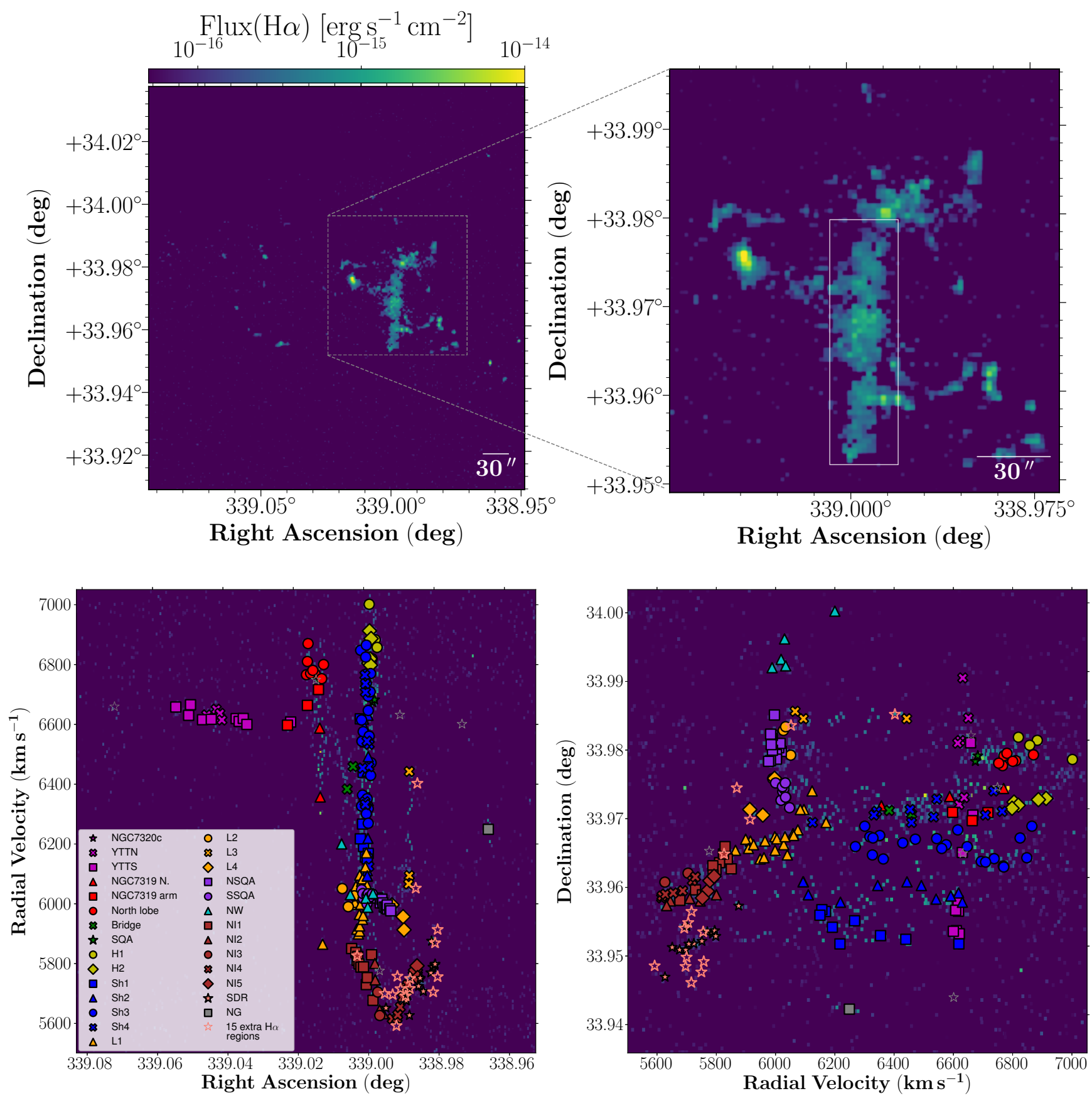

Fig. 14. $\mathrm{H} \alpha$ flux spatial map of entire SQ (upper left panel), $\mathrm{H} \alpha$ flux spatial map zoomed in to shock region (upper right panel). Lower left panel: radial velocity versus RA diagram and the lower right panel shows Dec versus radial velocity diagram. All the points in the lower panels of the figures have the same colours and markers as Fig. 7. The white rectangle centred on LSSR is overplotted with a solid line in the upper right panel. This rectangle is centred on the coordinate $\mathrm{RA}=339 \mathrm{deg}$ and Dec $=33.966 \mathrm{deg}$, with a width of $\Delta \mathrm{RA}=27.786$ arcsec and a height of $\Delta \mathrm{Dec}=99.512$ arcsec.

\subsection{The other galaxies in $S Q$}

In most articles that study SQ, the galaxies that are studied in detail are NGC 7317, NGC 7318a, NGC 7318b, and NGC 7319, as well as NGC 7320. In this work, thanks to the large SITELLE FoV, we were able to add to the study of the galaxy NGC 7320c (the OI) and the NG. In Figs. 15 and 16 we present the spatially resolved $\mathrm{H} \alpha$ flux and radial velocity maps for NG and NGC 7320c, respectively.

We detect $\mathrm{H} \alpha$ emission in NG. This, combined with the fact that NG is extended and presents a galactic rotation of $\pm 60 \mathrm{~km} \mathrm{~s}^{-1}$ as shown in Fig. 15 (e.g. Swaters et al. 2002, Westmoquette et al. 2013), indicates that NG is consistent with a dwarf galaxy that will end up interacting with the SQ system in the future, similar to M 82 (according to the Tully-Fisher relation). A more detailed study has been made for NG. In Fig. 15 we show the $\mathrm{H} \alpha$ flux (left panel) and the radial velocity map (right panel) of NG. The inner panel shows the Hubble Space Telescope optical imaging of NG. The velocity gradient is clearly seen from the south to the north $\left(v \sim 6300\right.$ to $\left.\sim 6150 \mathrm{~km} \mathrm{~s}^{-1}\right)$. As we can see in Table B.1, region 176, the average radial velocity for NG is $6246 \mathrm{~km} \mathrm{~s}^{-1}$. 

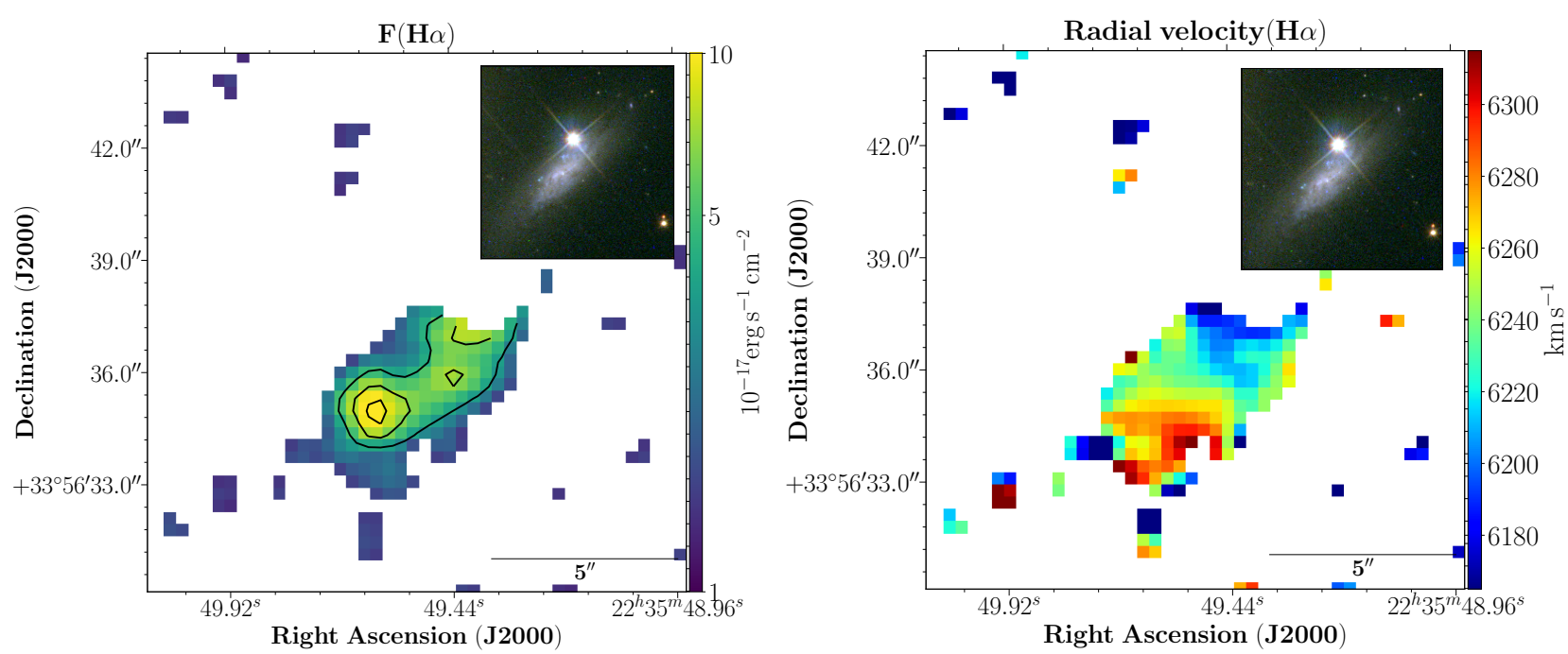

Fig. 15. NG $\mathrm{H} \alpha$ flux spatial map (left panel) and $\mathrm{H} \alpha$ radial velocity map (right panel). Inner panels: HST/WFC3 optical imaging for NG. The black contours are $1 \times 10^{-17}, 4 \times 10^{-17}$, and $7 \times 10^{-17} \mathrm{erg} \mathrm{s}^{-1} \mathrm{~cm}^{-2}$.
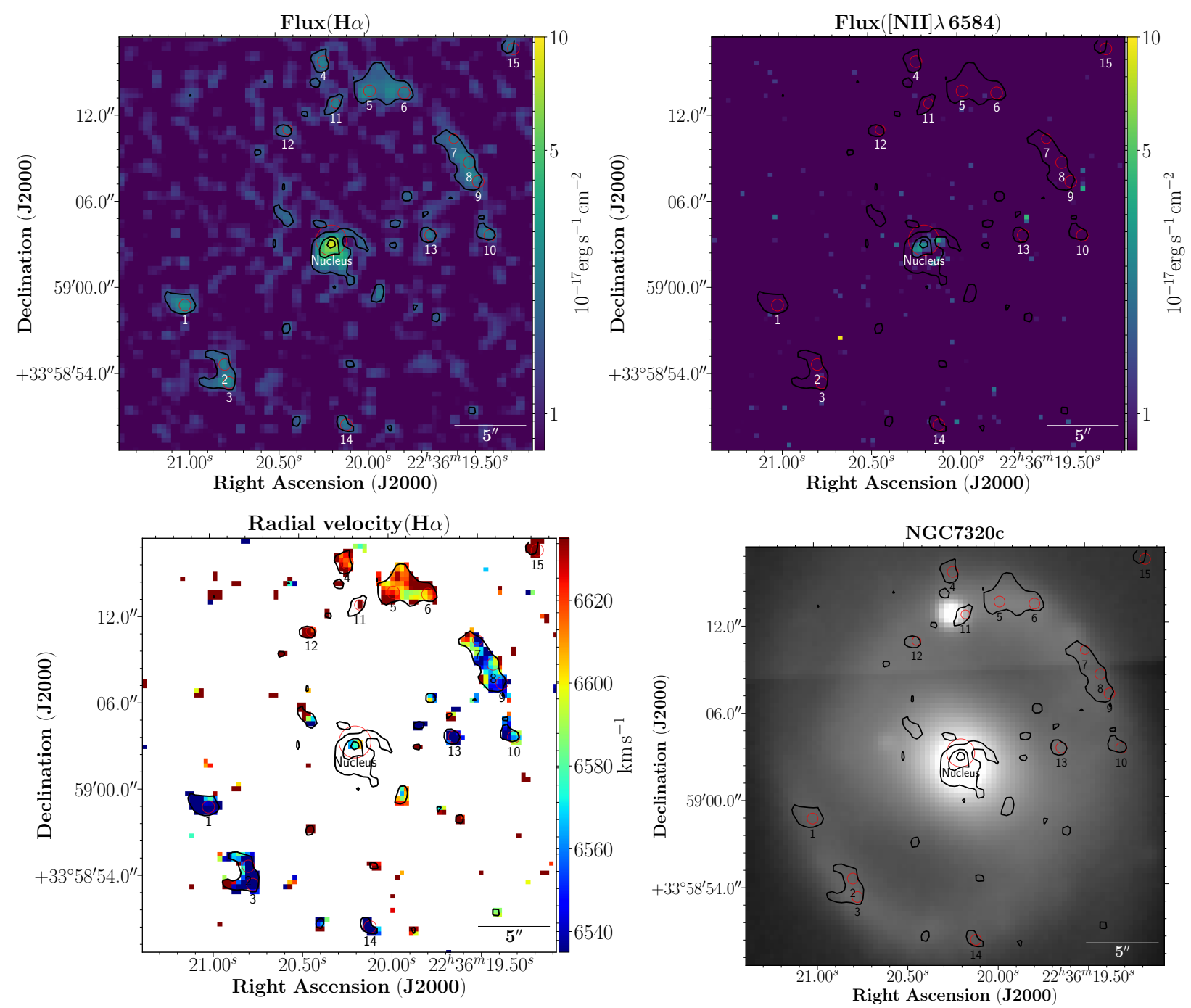

Fig. 16. NGC 7320c H $\alpha$ flux spatial map (upper left panel), [N II] 36584 flux spatial map (upper right panel), H $\alpha$ radial velocity map considering pixels with contrast $(\mathrm{H} \alpha) \geq 2.5$ (lower left panel), and deep image from SN2 filter (lower right panel). Red circles represent the locations of the NGC 7320c nucleus and $15 \mathrm{H} \alpha$ emission regions from its spiral arms. The black contours are $1.5 \times 10^{-17}, 4 \times 10^{-17}$, and $7 \times 10^{-17} \mathrm{erg} \mathrm{s}^{-1} \mathrm{~cm}^{-2}$. 


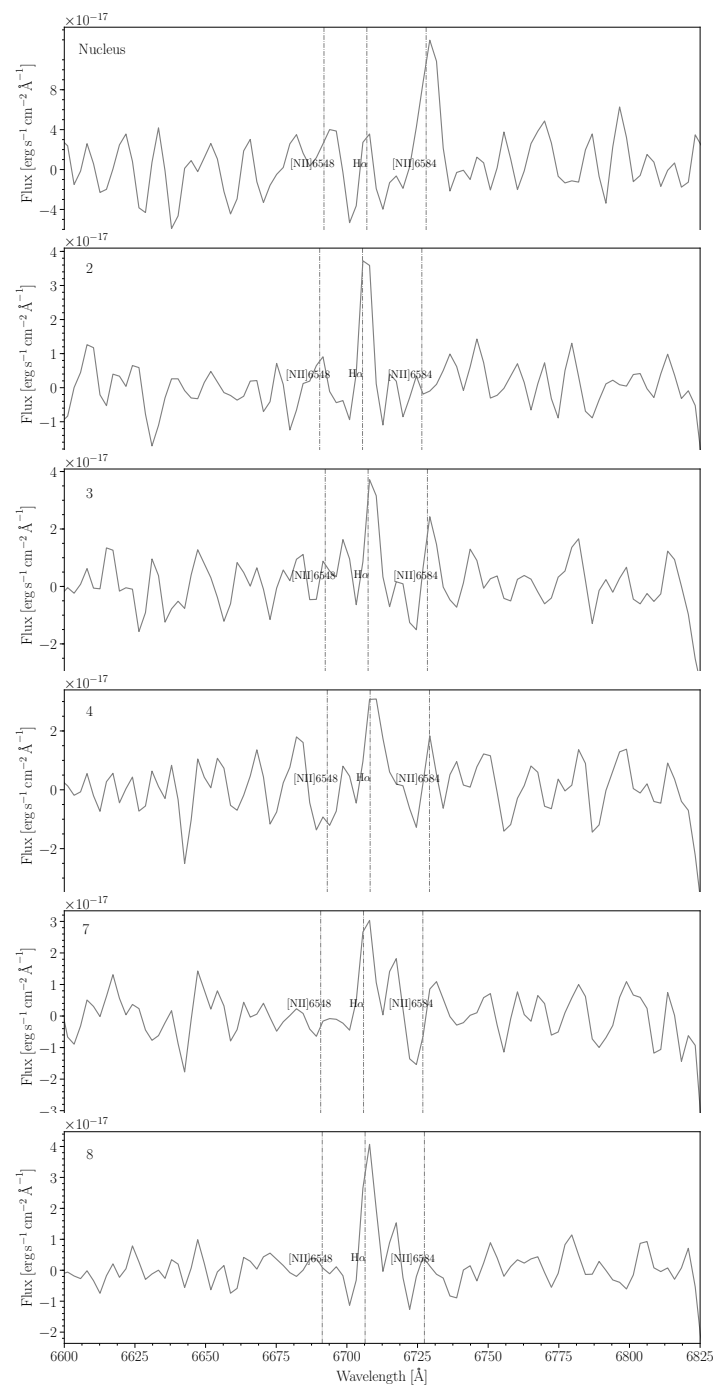

Fig. 17. Spectra from NGC 7320c nucleus, and several $\mathrm{H} \alpha$ regions in its galactic disc. Shown from top to bottom are the NGC 7320c galactic nucleus and regions 2, 3, 4, 7, and 8 (see Fig. 16). The black vertical dashed lines show the position of the emission lines studied for the SN3 filter at an $\mathrm{H} \alpha$ radial velocity of $\sim 6590 \mathrm{~km} \mathrm{~s}^{-1}$.

In Fig. 14 (upper left panel) we show the low surface brightness $\mathrm{H} \alpha$ emission in the strongly stripped galaxy NGC 7320c. Past interaction processes have stripped much of the gas in NGC 7320c. For a better understanding of this galaxy, we studied the $\mathrm{H} \alpha$ and [N II] $\lambda 6584$ fluxes for this galaxy. Figure 16 shows the $\mathrm{H} \alpha$ flux map (upper left panel), the [N II] $\lambda 6584$ flux map (upper right panel), the $\mathrm{H} \alpha$ radial velocity map (lower left panel), and the deep image from the SN2 filter (lower right panel) for NGC 7320c. In this figure, we see the presence of $\mathrm{H} \alpha$ and $[\mathrm{N}$ II $] \lambda 6584$ emission in the galactic nucleus, and $\mathrm{H} \alpha$ emission in several regions located in the spiral arms. Figure 17 shows examples of spectra for several regions with $\mathrm{H} \alpha$ emission found in NGC 7320c. Observing the fit for the galactic nucleus emission line (see upper panel in Fig. 17), we ascertain that the spectrum has an AGN profile (and found a strong emission of [N II] 26584). We barely observe [N II] $\lambda 6584$ emission (or it may be very small) in the rest of the regions located in the NGC 7320c spiral arm. The radial velocity map covers a range of radial velocities from $\sim 6540$ to $\sim 6640 \mathrm{~km} \mathrm{~s}^{-1}$.

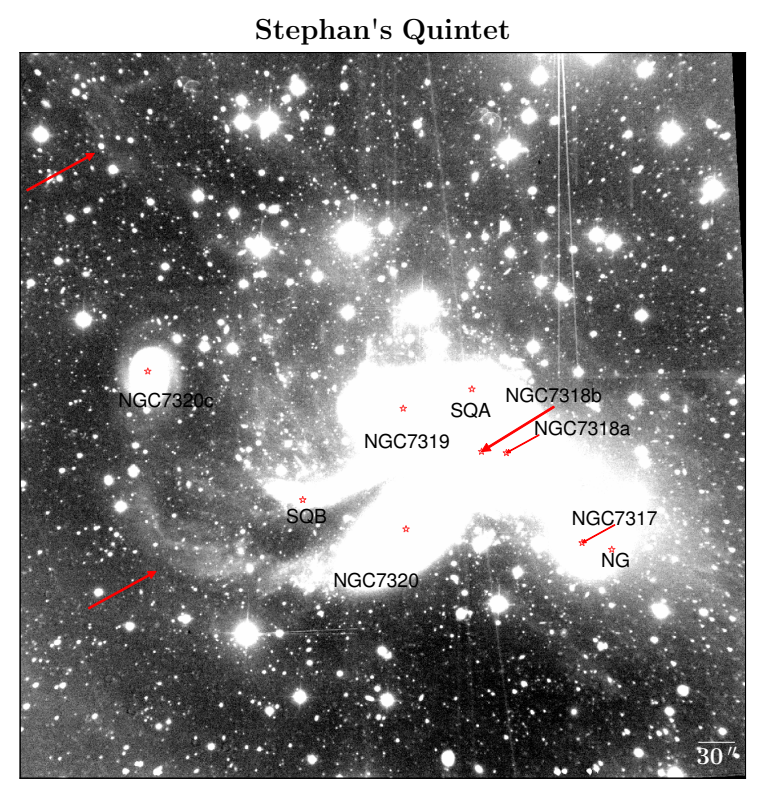

Fig. 18. Deep imaging from SN2 filter of SQ. This image displays the full extension of the entire system, including the diffuse ionised gas halo around the SQ. The most relevant components are indicated by their labels and red arrows. See the text for details.

\section{Discussion and final remarks}

A sample of $175 \mathrm{SQ} \mathrm{H} \alpha$ regions, 22 of them with two velocity components, has been made according to the following criteria: (i) the radial velocity of the region is within the range of SQ (between $\sim 5600$ and $\sim 7000 \mathrm{~km} \mathrm{~s}^{-1}$ ); (ii) the detection of at least one additional emission line besides $\mathrm{H} \alpha$. Additionally, in Appendix A we add the relevant information of 15 extra $\mathrm{H} \alpha$ emitters that we have found in the southern debris region, close to NI, and to the west of SQ (regions from 177 to 191 in Table A.1).

Two large groups of radial velocities were found (radial velocities $\leq 6160 \mathrm{~km} \mathrm{~s}^{-1}$ and $>6160 \mathrm{~km} \mathrm{~s}^{-1}$ ). We found five radial velocity components in SQ: (i) $v=[5600-5900] \mathrm{km} \mathrm{s}^{-1}$, associated with NI and SDR; (ii) $v=[5900-6100] \mathrm{km} \mathrm{s}^{-1}$, associated with the NSQA and SSQA and the strands that are connected to these zones; (iii) $v=[6100-6600] \mathrm{km} \mathrm{s}^{-1}$, associated with the strands located in the shock; (iv) $v=[6600-6800] \mathrm{km} \mathrm{s}^{-1}$, associated with YTT, SQA, NGC 7319, and the north lobe; and (v) $v=[6800-7000] \mathrm{km} \mathrm{s}^{-1}$, associated with the strands that connect the shock with SQA.

When considering a contrast $(\mathrm{H} \alpha) \geq 2$ and binning $6 \times 6$, we do not detect gas in the old tail, in the vicinity of NGC 7318A nor in NGC 7317. We also do not have evidence that there is any connection between (i) the north lobe in NGC 7319 and the left side of SQA; or (ii) the shock and the right part of NI nor SDR. Conversely, we find a link between the shock and the NGC 7319 AGN nucleus ( $\mathrm{H} \alpha$ bridge). This bridge was detected in other wavelengths (e.g. Iglesias-Páramo \& Vílchez 2001; Sulentic et al. 2001; Trinchieri et al. 2005; Cluver et al. 2010; Guillard et al. 2010, 2012; Appleton et al. 2013, 2017).

A region of remarkable line emission is located between NGC 7319 and the NI galaxy (NGC 7318B). A rectangular area has been defined (see Sect. 3.2, shown in Fig. 14) to include this emission which is associated with the LSSR. This has allowed us to investigate the complex kinematical nature of this emitting gas, making use of detailed spatially resolved multi-sincgaussian fitting. The LSSR has revealed a rich knotty 


\section{Stephan's Quintet}

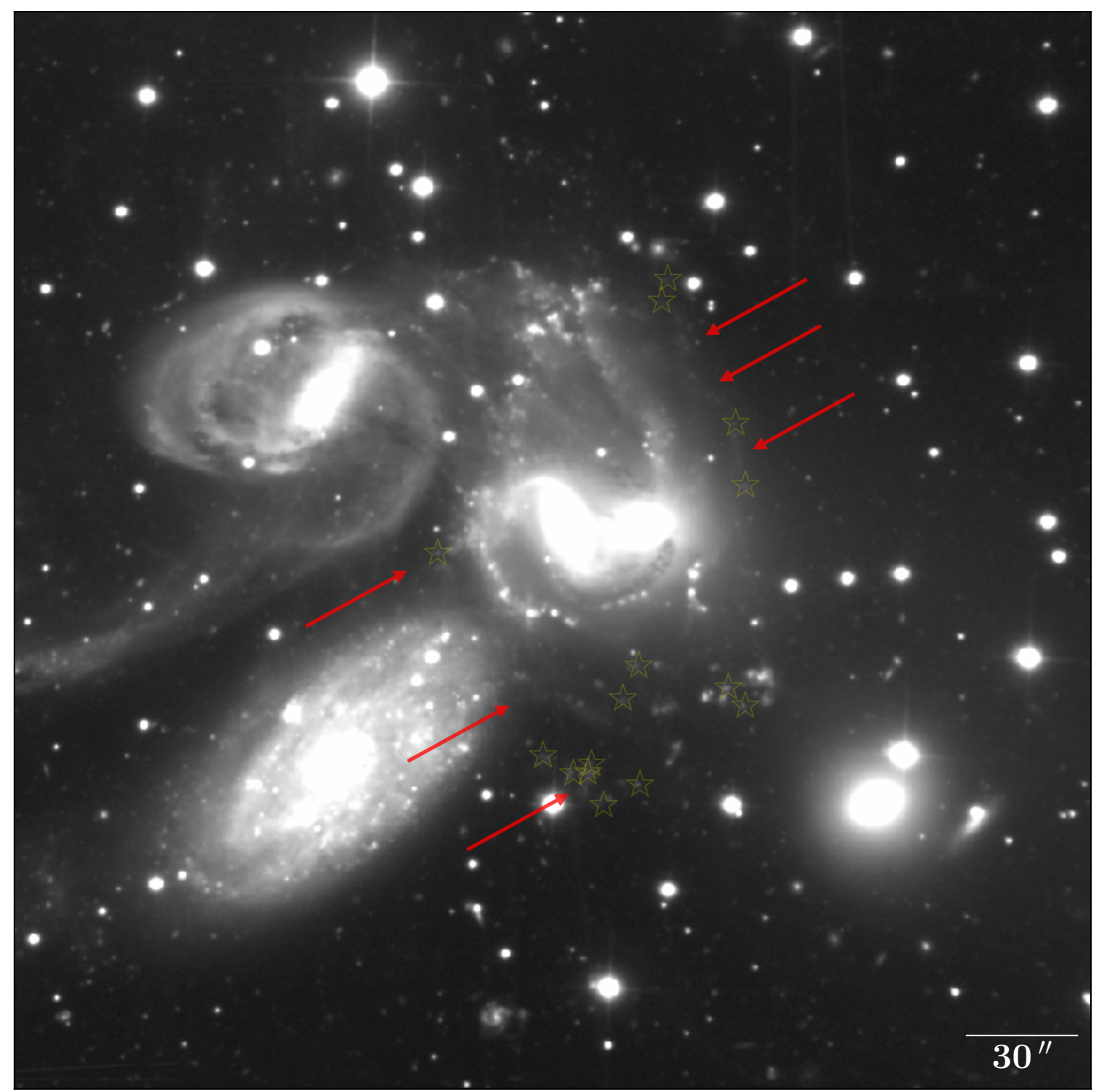

Fig. 19. Deep imaging from SN2 filter of SQ. Red arrows point out the possible outer structures that can be connected. The yellow stars show the location of the 15 extra $\mathrm{H} \alpha$ emitter regions presented in Table A.1.

substructure, spatially distributed, and also a set of kinematical properties of the $\mathrm{H} \alpha$ emitting knots. These knots show line profiles that have been fitted by two sincgaussian functions, with central velocities clustering around two main radial velocity values $\left(\sim 6000 \mathrm{~km} \mathrm{~s}^{-1}, \sim 6600 \mathrm{~km} \mathrm{~s}^{-1}\right)$, and a different broadness in each case. Overall, taking into account: (i) an oblique interaction over a large region, and new and preceding gas (e.g. O'Sullivan et al. 2009; Lesaffre et al. 2013; Appleton et al. 2017); and (ii) that the gas is excited by shocks (corresponding to a shock radial velocity $\sim 300 \mathrm{~km} \mathrm{~s}^{-1}$ according to Allen et al. 2008, see Duarte Puertas et al., in prep., Paper II), we suggest that a variety of interaction processes occurred previous to the intrusion of $\mathrm{NI}$ in the main group. We found sub-structures $\sim 400 \mathrm{pc}$ or larger in size, typical of giant HII extragalactic regions.

A possible outermost tail of $\mathrm{H} \alpha$ emitting regions is suggested here, which would be in agreement with Renaud et al. (2010). We provide an observational clue of this prediction. We found that this tidal tail connects with NSQA (reg 145). Other strands connected with NSQA have been found: (i) the L2 strand that connects NSQA and SSQA; (ii) the L3 strand that connects NSQA with region 161 up to radial velocities $\sim 6470 \mathrm{~km} \mathrm{~s}^{-1}$; and (iii) the L4 strand that connects NSQA with regions 154 and 155. This last connection could be extended up to the NI5 strand according to the diffuse gas show in the low surface brightness gas emission.

From our sample of SQ $\mathrm{H} \alpha$ emission regions we have studied the region 176 (i.e. NG) in detail (see Sect. 3.4). This region was previously observed by Torres-Flores et al. (2009; see their Fig. 14, ID 35) and by Rodríguez-Baras et al. (2014). In this paper we have made a spatially resolved study of the $\mathrm{H} \alpha$ radial velocity and $\mathrm{H} \alpha$ flux in this region. The data points out that this region is in fact a dwarf galaxy with a velocity gradient across its disc $\left( \pm 60 \mathrm{~km} \mathrm{~s}^{-1}\right.$, see Fig. 15), which was not considered by Rodríguez-Baras et al. (2014). These authors found an age of $\sim 10^{10} \mathrm{yr}$ for $80 \%$ of the stellar mass in this region, assuming that this region is a star cluster separated from a galaxy by some 
previous interactions. According to the Tully-Fisher relation, we found that NG would be similar to M 82. Additionally, we found $\mathrm{H} \alpha$ emission in the NGC 7320c galactic disc and a significant presence of broad [N II] $\lambda 6584$ emission in the NGC 7320c nucleus. This leads us to propose that NGC 7320c hosts an AGN (see Fig. 17).

A panoramic view of the full extent of the low surface brightness emission of the SQ is illustrated in Fig. 18, which shows a deep imaging of SQ obtained from the SITELLE SN2 filter. This figure displays the full extension and complexity of the entire system (including the diffuse ionised gas halo around the SQ, the old tail, the halo around NGC 7317, and a conspicuously low surface brightness extension to the north-east of the whole system). For details, the reader is referred to the recent work by Duc et al. (2018), which offers an interesting study of the complete system.

Figure 19 shows the deep image from the SN2 filter. In this figure, we suggest a possible tidal structure (delineated by an outer rim to the north-west of NGC 7318B/A) with diffuse gas emission that could connect the south part of L3 and NSQA with NI4, NI5, and SDR (marked by a green arrow). The detection of the 15 additional low surface brightness $\mathrm{H} \alpha$ emission regions (i.e. IDs from 177 to 191 in Table A.1, located in: SDR, close to NI, and to the west of SQ) with the expected radial velocities in this zone $\left(\sim 5600\right.$ to $\left.\sim 5900 \mathrm{~km} \mathrm{~s}^{-1}\right)$ has allowed us to support this possible connection. This outer rim is almost parallel with another inner rim (i.e. strand L4) that shows $\mathrm{H} \alpha$ emitting regions. Conversely, we do not discard the possibility of a connection between NI1 (i.e. region 38) and the left side of SDR going behind NGC 7320 (indicated by red arrows). Also this interaction could be supported by the existence of region $31\left(v \sim 5860 \mathrm{~km} \mathrm{~s}^{-1}\right)$ presenting the same radial velocity as the strand NI1 some $13 \mathrm{kpc}$ away to the east. The interaction suffered, which might explain these rims, appears complex, although we believe that it must have been produced by the interaction between NI and the group. The large-scale outer rim winding the NGC 7318B/A system clockwise north-west to south-east has been highlighted in continuum and from $\mathrm{H} \alpha$. This structure may be reminiscent of a previously proposed scenario for $\mathrm{SQ}$ as a sequence of individual interactions (Renaud et al. 2010). Further observations are needed with higher spectral resolution to distinguish the complex kinematics that is taking place in the LSSR.

Acknowledgements. We thank the anonymous referee for very constructive suggestions that have improved this manuscript. Based on observations obtained with SITELLE, a joint project of Université Laval, ABB, Université de Montréal, and the Canada-France-Hawaii Telescope (CFHT), which is operated by the National Research Council of Canada, the Institut National des Sciences de l'Univers of the Centre National de la Recherche Scientifique of France, and the University of Hawaii. The authors wish to recognise and acknowledge the very significant cultural role that the summit of Mauna Kea has always had within the indigenous Hawaiian community. We are most grateful to have the opportunity to conduct observations from this mountain. SDP, JIP, JVM, and CK acknowledge financial support from the Spanish Ministerio de Economía y Competitividad under grants AYA2013-47742-C4-1-P and AYA2016-79724C4-4-P, from Junta de Andalucía Excellence Project PEX2011-FQM-7058, and also acknowledge support from the State Agency for Research of the Spanish MCIU through the "Center of Excellence Severo Ochoa" award for the Instituto de Astrofísica de Andalucía (SEV-2017-0709). LD is grateful to the Natural Sciences and Engineering Research Council of Canada, the Fonds de Recherche du Québec, and the Canada Foundation for Innovation for funding. This research made use of Python (http: //www . python .org) and IPython (Pérez \& Grange 2007); Numpy (Van Der Walt et al. 2011); Pandas (McKinney 2010); of Matplotlib (Hunter 2007), a suite of open-source Python modules that provides a framework for creating scientific plots. This research made use of Astropy, a community-developed core Python package for Astronomy (Astropy Collaboration 2013). The Astropy web site is http://www .astropy.org. This research made use of astrodendro, a Python package to compute dendrograms of astronomical data (http: //www . dendrograms .org/)

\section{References}

Allen, R. J., \& Hartsuiker, J. W. 1972, Nature, 239, 324

Allen, M. G., Groves, B. A., Dopita, M. A., Sutherland, R. S., \& Kewley, L. J. 2008, ApJS, 178, 20

Appleton, P. N., Xu, K. C., Reach, W., et al. 2006, ApJ, 639, L51

Appleton, P. N., Guillard, P., Boulanger, F., et al. 2013, ApJ, 777, 66

Appleton, P. N., Guillard, P., Togi, A., et al. 2017, ApJ, 836, 76

Astropy Collaboration (Robitaille, T. P., et al.) 2013, A\&A, 558, A33

Burbidge, E. M., \& Burbidge, G. R. 1961, ApJ, 134, 244

Cluver, M. E., Appleton, P. N., Boulanger, F., et al. 2010, ApJ, 710, 248

de Mello, D. F., Urrutia-Viscarra, F., Mendes de Oliveira, C., et al. 2012, MNRAS, 426, 2441

Drissen, L., Martin, T., Rousseau-Nepton, L., et al. 2019, MNRAS, 485, 3930

Duc, P.-A., Cuillandre, J.-C., \& Renaud, F. 2018, MNRAS, 475, L40

Fedotov, K., Gallagher, S. C., Konstantopoulos, I. S., et al. 2011, AJ, 142, 42

Gallagher, S. C., Charlton, J. C., Hunsberger, S. D., Zaritsky, D., \& Whitmore, B. C. 2001, AJ, 122, 163

Grandmont, F., Drissen, L., Mandar, J., Thibault, S., \& Baril, M. 2012, in Ground-based and Airborne Instrumentation for Astronomy IV, SPIE Conf. Ser., 8446, 84460U

Guillard, P., Boulanger, F., Pineau Des Forêts, G., \& Appleton, P. N. 2009, A\&A, 502,515

Guillard, P., Boulanger, F., Cluver, M. E., et al. 2010, A\&A, 518, A59

Guillard, P., Boulanger, F., Pineau des Forêts, G., et al. 2012, ApJ, 749, 158

Heida, M., Jonker, P. G., Torres, M. A. P., \& Mineo, S. 2012, MNRAS, 424, 1563

Hunter, J. D. 2007, Comput. Sci. Eng., 9, 90

Hwang, J.-S., Struck, C., Renaud, F., \& Appleton, P. N. 2012, MNRAS, 419, 1780

Iglesias-Páramo, J., \& Vílchez, J. M. 2001, ApJ, 550, 204

Iglesias-Páramo, J., López-Martín, L., Vílchez, J. M., Petropoulou, V., \& Sulentic, J. W. 2012, A\&A, 539, A127

Konstantopoulos, I. S., Appleton, P. N., Guillard, P., et al. 2014, ApJ, 784, 1

Lesaffre, P., Pineau des Forêts, G., Godard, B., et al. 2013, A\&A, 550, A106

Lisenfeld, U., Braine, J., Duc, P.-A., et al. 2002, A\&A, 394, 823

Lisenfeld, U., Braine, J., Duc, P.-A., et al. 2004, A\&A, 426, 471

Martin, T., Drissen, L., \& Joncas, G. 2015, in Astronomical Data Analysis Software an Systems XXIV (ADASS XXIV), eds. A. R. Taylor, \& E. Rosolowsky, ASP Conf. Ser., 495, 327

Martin, T. B., Prunet, S., \& Drissen, L. 2016, MNRAS, 463, 4223

Martin, T. B., Drissen, L., \& Melchior, A.-L. 2018, MNRAS, 473, 4130

McKinney, W. 2010, in Proceedings of the 9th Python in Science Conference, eds. S. van der Walt, \& J. Millman, 51

Mendes de Oliveira, C., Plana, H., Amram, P., Balkowski, C., \& Bolte, M. 2001, AJ, 121, 2524

Mendes de Oliveira, C., Cypriano, E. S., Sodré, Jr., L., \& Balkowski, C. 2004, ApJ, 605, L17

Moles, M., Sulentic, J. W., \& Márquez, I. 1997, ApJ, 485, L69

Moles, M., Marquez, I., \& Sulentic, J. W. 1998, A\&A, 334, 473

Ohyama, Y., Nishiura, S., Murayama, T., \& Taniguchi, Y. 1998, ApJ, 492, L25

O'Sullivan, E., Giacintucci, S., Vrtilek, J. M., Raychaudhury, S., \& David, L. P. 2009, ApJ, 701, 1560

Pérez, F., \& Granger, B. E. 2007, Comput. Sci. Eng., 9, 21

Renaud, F., Appleton, P. N., \& Xu, C. K. 2010, ApJ, 724, 80

Rodríguez-Baras, M., Rosales-Ortega, F. F., Díaz, A. I., Sánchez, S. F., \& Pasquali, A. 2014, MNRAS, 442, 495

Stephan, M. 1877, MNRAS, 37, 334

Sulentic, J. W., Rosado, M., Dultzin-Hacyan, D., et al. 2001, AJ, 122, 2993

Swaters, R. A., van Albada, T. S., van der Hulst, J. M., \& Sancisi, R. 2002, A\&A, 390,829

Torres-Flores, S., Mendes de Oliveira, C., de Mello, D. F., et al. 2009, A\&A, 507,723

Trancho, G., Konstantopoulos, I. S., Bastian, N., et al. 2012, ApJ, 748, 102

Trinchieri, G., Sulentic, J., Breitschwerdt, D., \& Pietsch, W. 2003, A\&A, 401, 173

Trinchieri, G., Sulentic, J., Pietsch, W., \& Breitschwerdt, D. 2005, A\&A, 444, 697

Van Der Walt, S., Colbert, S. C., \& Varoquaux, G. 2011, Comput. Sci. Eng., 13, 22

Westmoquette, M. S., Smith, L. J., Gallagher, J. S., \& Walter, F. 2013, MNRAS, 428, 1743

Williams, B. A., Yun, M. S., \& Verdes-Montenegro, L. 2002, AJ, 123, 2417

Xu, C., Sulentic, J. W., \& Tuffs, R. 1999, ApJ, 512, 178

Xu, C. K., Iglesias-Páramo, J., Burgarella, D., et al. 2005, ApJ, 619, L95 


\section{Appendix A: $15 \mathrm{H} \alpha$ emitters in outer debris regions}

A close visual inspection, guided by the HST/WFC3 images (PID 11502, PI Keith S. Noll) of the SQ outskirts of our deep SITELLE images has allowed us to define a set of 15 extra $\mathrm{H} \alpha$ regions (located in SDR, near NI, and west of SQ). In Table A.1 we show the relevant information for these $\mathrm{H} \alpha$ emitters for the regions from ID 177 to 191 .

Table A.1. Positional information of 15 extra $\mathrm{H} \alpha$ emitter regions in the southern debris region, close to the NI, and to the west of SQ.

\begin{tabular}{|c|c|c|c|}
\hline \multirow{2}{*}{$\begin{array}{l}(1) \\
\text { Region } \\
\text { ID }\end{array}$} & \multicolumn{2}{|c|}{ (2) } & \multirow{2}{*}{$\begin{array}{c}(3) \\
\text { Velocity } \\
\left(\mathrm{km} \mathrm{s}^{-1}\right)\end{array}$} \\
\hline & $\begin{array}{c}\alpha \\
(\mathrm{h} \mathrm{m} \mathrm{s})\end{array}$ & $\begin{array}{l}\delta \\
\left({ }^{\circ}, \prime \prime\right)\end{array}$ & \\
\hline 177 & 223558.8 & 335655.4 & 5698 \\
\hline 178 & 223558.1 & 335650.5 & 5695 \\
\hline 179 & 223557.8 & 335653.0 & 5756 \\
\hline 180 & 223557.8 & 335650.8 & 5590 \\
\hline 181 & 223557.5 & 335642.3 & 5714 \\
\hline 182 & 223556.7 & 335647.5 & 5748 \\
\hline 183 & 223557.1 & 335710.0 & 5690 \\
\hline 184 & 223556.7 & 335718.5 & 5714 \\
\hline 185 & 223554.8 & 335712.5 & 5703 \\
\hline 186 & 223554 & 33577.7 & 5754 \\
\hline 187 & 223554. & 33585.7 & 5912 \\
\hline 188 & 223556. & 33590.9 & 6401 \\
\hline 189 & 223556.1 & 335855.1 & 6051 \\
\hline 190 & 223554.6 & 335822.1 & 5867 \\
\hline 191 & 22361.0 & 35749.0 & 5824 \\
\hline
\end{tabular}

Notes. The columns correspond to: (1) identifier of the $\mathrm{H} \alpha$ emission regions (ID); (2) right ascension (hours, minutes, and seconds) and declination (degrees, arcminutes, and arcseconds); (3) velocity $\left(\mathrm{km} \mathrm{s}^{-1}\right)$. Information about the 15 extra $\mathrm{H} \alpha$ emitter regions is given in SDR, close to the NI, and to the west of SQ.

\section{Appendix B: Additional table}

Table B.1. Relevant information of $\mathrm{H} \alpha$ emission regions in Stephan's Quintet.

\begin{tabular}{|c|c|c|c|c|}
\hline \multirow{2}{*}{$\begin{array}{c}(1) \\
\text { Region } \\
\text { ID }\end{array}$} & (2) & \multirow{2}{*}{$\begin{array}{c}(3) \\
\text { Velocity } \\
\left(\mathrm{km} \mathrm{s}^{-1}\right)\end{array}$} & \multirow{2}{*}{$\begin{array}{c}(4) \\
\text { Area } \\
\left(\mathrm{kpc}^{2}\right) \\
\end{array}$} & \multirow{2}{*}{$\begin{array}{c}(5) \\
\text { Subzone }\end{array}$} \\
\hline & $\begin{array}{c}\alpha \\
(\mathrm{h} \mathrm{m} \mathrm{s}) \\
\end{array}$ & & & \\
\hline $1^{(\dagger)}$ & $223620.3+335904.4$ & $6657 \pm 24$ & - & NGC 7320c \\
\hline 2 & $223615.3+33591.9$ & $6644 \pm 3$ & 1.0 & YTT \\
\hline 3 & $223615.2+335859.9$ & $6656 \pm 6$ & 0.6 & YTT \\
\hline 4 & $223614.1+33581.0$ & $6628 \pm 2$ & 1.3 & YTT \\
\hline 5 & $223614.0+335821.4$ & $6664 \pm 11$ & 0.2 & YTT \\
\hline 6 & $223613.0+335736.5$ & $6613 \pm 5$ & 0.5 & YTT \\
\hline 7 & $223612.6+335933.3$ & $6630 \pm 15$ & 0.2 & YTT \\
\hline 8 & $223612.4+335730.2$ & $6614 \pm 4$ & 1.3 & YTT \\
\hline 9 & $223611.8+335911.5$ & $6648 \pm 3$ & 1.1 & YTT \\
\hline 10 & $223611.5+335859.6$ & $6622 \pm 1$ & 1.3 & YTT \\
\hline 11 & $223611.5+335826.0$ & $6618 \pm 5$ & 0.5 & YTT \\
\hline 12 & $223611.5+335830.3$ & $6635 \pm 6$ & 0.3 & YTT \\
\hline 13 & $223611.4+335858.6$ & $6612 \pm 1$ & 1.3 & YTT \\
\hline 14 & $223610.2+335718.5$ & $6618 \pm 2$ & 2.3 & YTT \\
\hline 15 & $223610.0+335720.0$ & $6608 \pm 1$ & 1.7 & YTT \\
\hline 16 & $22369.6+335717.7$ & $6618 \pm 5$ & 0.3 & YTT \\
\hline 17 & $22369.4+335719.2$ & $6598 \pm 4$ & 0.8 & YTT \\
\hline 18 & $22366.0+335820.0$ & $6595 \pm 7$ & 0.3 & NGC 7319 “Arm" \\
\hline 19 & $22365.7+335729.9$ & $6606 \pm 5$ & 0.5 & YTT \\
\hline 20 & $22364.4+335844.9$ & $6764 \pm 4$ & 1.1 & North lobe \\
\hline 21 & $22364.3+335846.8$ & $6809 \pm 4$ & 1.2 & North lobe \\
\hline 22 & $22364.3+335816.0$ & $6662 \pm 17$ & 0.2 & NGC 7319 "Arm" \\
\hline 23 & $22364.3+335850.4$ & $6868 \pm 6$ & 0.7 & North lobe \\
\hline 24 & $22364.0+335850.0$ & $6769 \pm 7$ & 1.3 & North lobe \\
\hline 25 & $22363.8+335851.3$ & $6779 \pm 5$ & 0.9 & North lobe \\
\hline 26 & $22363.4+335832.3$ & $6768 \pm 9$ & 3.8 & NGC 7319 Nucl. \\
\hline 27 & $22363.3+335828.4$ & $6586 \pm 20$ & 3.9 & NGC 7319 Nucl. \\
\hline 28 & $22363.3+335820.6$ & $6715 \pm 7$ & 0.5 & NGC 7319 "Arm" \\
\hline 29 & $22363.3+335823.5$ & $6355 \pm 11$ & 0.3 & NGC 7319 Nucl. \\
\hline 30 & $22363.1+335845.5$ & $6750 \pm 8$ & 0.5 & North lobe \\
\hline 31 & $22363.1+33580.1$ & $5863 \pm 12$ & 0.2 & L1 \\
\hline 32 & $22362.9+335846.8$ & $6799 \pm 12$ & 0.2 & North lobe \\
\hline 33 & $22361.4+335849.0$ & $6049 \pm 7$ & 0.5 & L2 \\
\hline 34 & $22361.4+3405.7$ & $6198 \pm 14$ & 0.2 & NW \\
\hline 35 & $22361.0+335821.0$ & $6381 \pm 10$ & 1.0 & Bridge \\
\hline 36 & $22360.9+335836.8$ & $5988 \pm 13$ & 0.2 & L2 \\
\hline 37 & $22360.7+335950.7$ & $6028 \pm 6$ & 0.6 & NW \\
\hline 38 & $22360.6+335749.9$ & $5849 \pm 9$ & 0.3 & NI1 \\
\hline 39 & $22360.5+33586.3$ & $5996 \pm 7$ & 0.6 & L1 \\
\hline 40 & $22360.6+335817.9$ & $6457 \pm 14$ & 0.3 & Bridge \\
\hline 41 & $22360.5+33592.1$ & $6024 \pm 8$ & 0.5 & L2 \\
\hline 42 & $22360.4+335755.3$ & $5835 \pm 4$ & 0.7 & NI1 \\
\hline 43 & $22360.4+33585.0$ & $6003 \pm 11$ & 0.2 & $\mathrm{~L} 1$ \\
\hline 44 & $22360.4+33588.2$ & $6057 \pm 12$ & 0.2 & $\mathrm{~L} 1$ \\
\hline 45 & $22360.3+335752.7$ & $5809 \pm 6$ & 1.0 & NI1 \\
\hline 46 & $22360.2+335757.5$ & $5822 \pm 5$ & 0.8 & NI1 \\
\hline 47 & $22360.2+33584.0$ & $5913 \pm 9$ & 0.2 & $\mathrm{~L} 1$ \\
\hline 48 & $22360.2+33585.3$ & $5898 \pm 12$ & 0.3 & L1 \\
\hline 49 & $22360.2+335748.8$ & $5797 \pm 10$ & 0.6 & NI1 \\
\hline
\end{tabular}

Notes. The columns correspond to: (1) identifier of the $\mathrm{H} \alpha$ emission regions (ID); (2) right ascension (hours, minutes, and seconds) and declination (degrees, arcminutes, and arcseconds); (3) $\mathrm{H} \alpha$ radial velocity $\left(\mathrm{km} \mathrm{s}^{-1}\right)$; (4) area $\left(\mathrm{kpc}^{2}\right)$. (5) Subzone. "( $\left.\dagger\right)$ " (ID 1) denotes the information about NGC 7320c. For regions with two velocity components we use " $(a)$ " and " $(b)$ " for low- and high-velocity components, respectively. 
Table B.1. continued.

\begin{tabular}{|c|c|c|c|c|}
\hline (1) & (2) & (3) & (4) & (5) \\
\hline $\begin{array}{l}\text { Region } \\
\text { ID }\end{array}$ & $\begin{array}{c}\alpha \\
(\mathrm{h} \mathrm{m} \mathrm{s})\end{array}$ & $\begin{array}{l}\text { Velocity } \\
\left(\mathrm{km} \mathrm{s}^{-1}\right)\end{array}$ & $\begin{array}{c}\text { Area } \\
\left(\mathrm{kpc}^{2}\right)\end{array}$ & Subzone \\
\hline 50 & $22360.0+33584.2$ & $5963 \pm 10$ & 0.3 & L1 \\
\hline $51^{(a)}$ & $223560.0+335711.1$ & $6215 \pm 50$ & 1.9 & Sh1 \\
\hline $51^{(b)}$ & $223560.0+335711.1$ & $6617 \pm 20$ & 1.9 & Sh1 \\
\hline 52 & $223559.9+335751.4$ & $5786 \pm 3$ & 1.7 & NI1 \\
\hline 53 & $22360.0+33580.4$ & $5907 \pm 10$ & 0.2 & L1 \\
\hline 54 & $22360.1+33582.3$ & $5924 \pm 10$ & 0.2 & L1 \\
\hline 55 & $22360.0+335820.7$ & $6093 \pm 11$ & 0.2 & L1 \\
\hline 56 & $223560.0+335755.5$ & $6846 \pm 13$ & 0.2 & Sh3 \\
\hline 57 & $223560.0+335759.1$ & $5997 \pm 18$ & 0.2 & L1 \\
\hline $58^{(a)}$ & $223559.9+335757.5$ & $6037 \pm 14$ & 0.6 & L1 \\
\hline $58^{(b)}$ & $223559.9+335757.5$ & $6325 \pm 30$ & 0.6 & Sh3 \\
\hline $59^{(a)}$ & $223559.9+33585.2$ & $6165 \pm 16$ & 1.6 & L1 \\
\hline $59^{(b)}$ & $223559.9+33585.2$ & $6621 \pm 32$ & 1.6 & Sh3 \\
\hline $60^{(a)}$ & $223559.9+335810.0$ & $6073 \pm 14$ & 0.3 & L1 \\
\hline $60^{(b)}$ & $223559.9+335810.0$ & $6612 \pm 17$ & 0.3 & Sh3 \\
\hline 61 & $223559.9+335821.7$ & $6046 \pm 12$ & 0.2 & SSQA \\
\hline 62 & $223559.9+335713.3$ & $6438 \pm 10$ & 0.7 & Sh1 \\
\hline 63 & $223559.8+335722.8$ & $6265 \pm 21$ & 0.2 & Sh1 \\
\hline 64 & $223559.8+335736.6$ & $6616 \pm 11$ & 0.6 & Sh2 \\
\hline 65 & $223559.7+335745.8$ & $5787 \pm 5$ & 1.1 & NI1 \\
\hline $66^{(a)}$ & $223559.8+335754.5$ & $5946 \pm 12$ & 0.5 & L1 \\
\hline $66^{(b)}$ & $223559.8+335754.5$ & $6362 \pm 14$ & 0.5 & Sh3 \\
\hline 67 & $223559.8+335755.5$ & $5955 \pm 13$ & 0.5 & $\mathrm{~L} 1$ \\
\hline $68^{(a)}$ & $223559.7+33581.0$ & $6025 \pm 9$ & 1.6 & L1 \\
\hline $68^{(b)}$ & $223559.7+33581.0$ & $6574 \pm 37$ & 1.6 & Sh3 \\
\hline $69^{(a)}$ & $223559.8+335820.7$ & $6081 \pm 20$ & 0.3 & L1 \\
\hline $69^{(b)}$ & $223559.8+335820.7$ & $6452 \pm 32$ & 0.3 & Sh4 \\
\hline 70 & $223559.7+335825.5$ & $6016 \pm 4$ & 0.7 & SSQA \\
\hline 71 & $223559.7+335827.4$ & $6032 \pm 8$ & 0.4 & SSQA \\
\hline 72 & $223559.8+335718.7$ & $6189 \pm 15$ & 0.3 & Sh1 \\
\hline 73 & $223559.7+335713.9$ & $6352 \pm 10$ & 0.7 & Sh1 \\
\hline 74 & $223559.7+335735.6$ & $6589 \pm 10$ & 0.7 & Sh2 \\
\hline 75 & $223559.6+335738.1$ & $5728 \pm 5$ & 1.5 & NI1 \\
\hline $76^{(a)}$ & $223559.7+335819.7$ & $6312 \pm 27$ & 0.6 & Sh4 \\
\hline $76^{(b)}$ & $223559.7+335819.7$ & $6761 \pm 35$ & 0.6 & Sh4 \\
\hline 77 & $223559.6+335735.2$ & $6540 \pm 12$ & 0.7 & Sh2 \\
\hline 78 & $223559.6+335728.5$ & $6154 \pm 13$ & 0.2 & Sh1 \\
\hline $79^{(a)}$ & $223559.5+33585.1$ & $6326 \pm 31$ & 1.3 & Sh3 \\
\hline $79^{(b)}$ & $223559.5+33585.1$ & $6823 \pm 10$ & 1.3 & Sh3 \\
\hline $80^{(a)}$ & $223559.6+335817.4$ & $6343 \pm 19$ & 0.3 & Sh4 \\
\hline $80^{(b)}$ & $223559.6+335817.4$ & $6752 \pm 23$ & 0.3 & Sh4 \\
\hline $81^{(a)}$ & $223559.5+335830.3$ & $6123 \pm 17$ & 0.3 & L1 \\
\hline $81^{(b)}$ & $223559.5+335830.3$ & $6734 \pm 26$ & 0.3 & Sh4 \\
\hline 82 & $223559.5+335939.6$ & $6017 \pm 4$ & 1.0 & NW \\
\hline $83^{(a)}$ & $223559.4+335733.3$ & $6211 \pm 22$ & 3.0 & Sh2 \\
\hline $83^{(b)}$ & $223559.4+335733.3$ & $6626 \pm 11$ & 3.0 & $\operatorname{Sh} 2$ \\
\hline 84 & $223559.5+335750.6$ & $6767 \pm 12$ & 0.3 & Sh3 \\
\hline 85 & $223559.5+335755.4$ & $6735 \pm 10$ & 0.3 & Sh3 \\
\hline $86^{(a)}$ & $223559.4+33587.0$ & $6059 \pm 13$ & 0.8 & L1 \\
\hline $86^{(b)}$ & $223559.4+33587.0$ & $6350 \pm 25$ & 0.8 & Sh3 \\
\hline $87^{(a)}$ & $223559.5+335811.5$ & $6299 \pm 21$ & 0.3 & Sh3 \\
\hline $87^{(b)}$ & $223559.5+335811.5$ & $6863 \pm 16$ & 0.3 & Sh3 \\
\hline 88 & $223559.5+335813.8$ & $6168 \pm 18$ & 0.2 & L1 \\
\hline $89^{(a)}$ & $223559.5+335826.4$ & $6542 \pm 22$ & 0.7 & Sh4 \\
\hline $89^{(b)}$ & $223559.5+335826.4$ & $6861 \pm 36$ & 0.7 & Sh4 \\
\hline 90 & $223559.4+335829.9$ & $6022 \pm 5$ & 0.8 & SSQA \\
\hline 91 & $223559.4+335831.3$ & $6026 \pm 10$ & 0.4 & SSQA \\
\hline 92 & $223559.5+335834.2$ & $5999 \pm 7$ & 0.5 & SSQA \\
\hline 93 & $223559.4+335725.3$ & $6148 \pm 11$ & 0.2 & Sh1 \\
\hline
\end{tabular}

Table B.1. continued.

\begin{tabular}{|c|c|c|c|c|}
\hline \multirow{2}{*}{$\begin{array}{c}(1) \\
\text { Region } \\
\text { ID }\end{array}$} & (2) & \multirow{2}{*}{$\begin{array}{c}(3) \\
\text { Velocity } \\
\left(\mathrm{km} \mathrm{s}^{-1}\right)\end{array}$} & \multirow{2}{*}{$\begin{array}{c}(4) \\
\text { Area } \\
\left(\mathrm{kpc}^{2}\right) \\
\end{array}$} & \multirow{2}{*}{$\begin{array}{c}(5) \\
\text { Subzone }\end{array}$} \\
\hline & $\begin{array}{c}\alpha \\
(\mathrm{h} \mathrm{m} \mathrm{s})\end{array}$ & & & \\
\hline $94^{(a)}$ & $223559.5+335735.9$ & $5749 \pm 15$ & 0.3 & NI1 \\
\hline $94^{(b)}$ & $223559.5+335735.9$ & $6049 \pm 28$ & 0.3 & $\operatorname{Sh} 2$ \\
\hline $95^{(a)}$ & $223559.4+335742.9$ & $6091 \pm 57$ & 0.3 & Sh2 \\
\hline $95^{(b)}$ & $223559.4+335742.9$ & $6442 \pm 27$ & 0.3 & Sh2 \\
\hline 96 & $223559.3+335824.4$ & $6803 \pm 11$ & 0.8 & $\mathrm{H} 2$ \\
\hline 97 & $223559.2+335934.6$ & $5986 \pm 5$ & 0.6 & NW \\
\hline 98 & $223559.3+335827.0$ & $6031 \pm 7$ & 0.4 & SSQA \\
\hline 99 & $223559.4+335727.5$ & $6166 \pm 23$ & 0.2 & Sh1 \\
\hline $100^{(a)}$ & $223559.4+335741.0$ & $6233 \pm 27$ & 0.3 & Sh3 \\
\hline $100^{(b)}$ & $223559.4+335741.0$ & $6490 \pm 34$ & 0.3 & $\mathrm{Sh} 2$ \\
\hline 101 & $223559.4+335753.2$ & $6692 \pm 16$ & 0.2 & Sh3 \\
\hline $102^{(a)}$ & $223559.3+33581.2$ & $6267 \pm 17$ & 0.3 & Sh3 \\
\hline $102^{(b)}$ & $223559.3+33581.2$ & $6644 \pm 43$ & 0.3 & Sh3 \\
\hline $103^{(a)}$ & $223559.3+33585.7$ & $6030 \pm 13$ & 0.5 & L1 \\
\hline $103^{(b)}$ & $223559.3+33585.7$ & $6316 \pm 42$ & 0.5 & Sh3 \\
\hline $104^{(a)}$ & $223559.4+335813.4$ & $6124 \pm 72$ & 1.6 & $\mathrm{Sh} 4$ \\
\hline $104^{(b)}$ & $223559.4+335813.4$ & $6460 \pm 31$ & 1.6 & Sh4 \\
\hline 105 & $223559.2+335825.7$ & $6911 \pm 11$ & 0.6 & $\mathrm{H} 2$ \\
\hline 106 & $223559.2+335832.2$ & $6021 \pm 8$ & 0.7 & SSQA \\
\hline 107 & $223559.2+335753.1$ & $6707 \pm 13$ & 0.8 & $\operatorname{Sh} 3$ \\
\hline 108 & $223559.2+33580.2$ & $6558 \pm 27$ & 0.2 & Sh3 \\
\hline 109 & $223559.2+335816.3$ & $6532 \pm 19$ & 0.2 & Sh4 \\
\hline 110 & $223559.2+335822.1$ & $6817 \pm 8$ & 0.8 & $\mathrm{H} 2$ \\
\hline 111 & $223559.2+335846.8$ & $7000 \pm 6$ & 0.5 & H1 \\
\hline 112 & $223559.1+33594.3$ & $6032 \pm 4$ & 1.9 & $\mathrm{~L} 2$ \\
\hline 113 & $223559.1+335749.9$ & $6768 \pm 9$ & 0.3 & Sh3 \\
\hline 114 & $223559.1+33583.1$ & $6427 \pm 14$ & 0.3 & Sh3 \\
\hline 115 & $223559.1+335821.1$ & $6797 \pm 13$ & 0.4 & $\mathrm{H} 2$ \\
\hline 116 & $223559.1+335825.4$ & $6885 \pm 8$ & 1.4 & $\mathrm{H} 2$ \\
\hline 117 & $223559.0+335733.2$ & $5675 \pm 1$ & 2.2 & NI1 \\
\hline 118 & $223559.1+33581.1$ & $5827 \pm 9$ & 0.3 & NI1 \\
\hline 119 & $223559.1+33585.0$ & $6469 \pm 18$ & 0.2 & Sh3 \\
\hline 120 & $223559.0+335834.1$ & $6031 \pm 3$ & 1.5 & SSQA \\
\hline 121 & $223559.0+335845.7$ & $6672 \pm 4$ & 3.0 & SQA \\
\hline 122 & $223558.8+335848.6$ & $6680 \pm 2$ & 4.9 & SQA \\
\hline 123 & $223558.9+335858.1$ & $6818 \pm 10$ & 0.2 & H1 \\
\hline 124 & $223558.8+335935.5$ & $6032 \pm 9$ & 0.3 & NW \\
\hline 125 & $223558.8+335736.0$ & $5799 \pm 11$ & 0.7 & NI2 \\
\hline 126 & $223558.6+335856.0$ & $6881 \pm 8$ & 1.1 & H1 \\
\hline 127 & $223558.8+335733.2$ & $5744 \pm 4$ & 1.4 & NI2 \\
\hline 128 & $223558.7+335734.1$ & $5708 \pm 3$ & 0.7 & NI2 \\
\hline 129 & $223558.4+335851.1$ & $6017 \pm 2$ & 2.3 & NSQA \\
\hline 130 & $223558.6+335747.5$ & $5703 \pm 3$ & 1.1 & NI3 \\
\hline 131 & $223558.6+335854.4$ & $6856 \pm 11$ & 0.6 & H1 \\
\hline 132 & $223558.4+335852.4$ & $6009 \pm 2$ & 1.4 & NSQA \\
\hline 133 & $223558.4+335732.8$ & $5643 \pm 1$ & 1.8 & NI2 \\
\hline 134 & $223558.4+335742.4$ & $5624 \pm 5$ & 1.0 & NI3 \\
\hline 135 & $223558.4+335848.5$ & $6001 \pm 6$ & 1.2 & NSQA \\
\hline 136 & $223558.1+335854.3$ & $6013 \pm 3$ & 1.8 & NSQA \\
\hline 137 & $223558.1+335846.5$ & $6001 \pm 3$ & 2.1 & NSQA \\
\hline 138 & $223558.2+335729.9$ & $5630 \pm 2$ & 1.6 & NI2 \\
\hline 139 & $223558.0+335859.1$ & $5987 \pm 2$ & 1.6 & NSQA \\
\hline 140 & $223557.9+335850.3$ & $5987 \pm 5$ & 1.4 & NSQA \\
\hline 141 & $223557.8+335856.5$ & $5984 \pm 2$ & 1.8 & NSQA \\
\hline 142 & $223557.8+335854.2$ & $5983 \pm 3$ & 1.5 & NSQA \\
\hline 143 & $223557.6+335845.1$ & $5980 \pm 3$ & 2.4 & NSQA \\
\hline 144 & $223558.0+33578.8$ & $5650 \pm 13$ & 0.2 & SDR \\
\hline 145 & $223557.7+33599.8$ & $5994 \pm 11$ & 0.2 & NSQA \\
\hline 146 & $223557.5+335859.6$ & $5981 \pm 2$ & 2.2 & NSQA \\
\hline
\end{tabular}


Table B.1. continued.

\begin{tabular}{|c|c|c|c|c|}
\hline \multirow{2}{*}{$\begin{array}{c}(1) \\
\text { Region } \\
\text { ID }\end{array}$} & (2) & \multirow{2}{*}{$\begin{array}{c}(3) \\
\text { Velocity } \\
\left(\mathrm{km} \mathrm{s}^{-1}\right)\end{array}$} & \multirow{2}{*}{$\begin{array}{c}(4) \\
\text { Area } \\
\left(\mathrm{kpc}^{2}\right)\end{array}$} & \multirow{2}{*}{$\begin{array}{c}(5) \\
\text { Subzone }\end{array}$} \\
\hline & $\begin{array}{cl}\alpha & \delta \\
(\mathrm{h} \mathrm{m} \mathrm{s}) & \left({ }^{\circ \prime \prime \prime}\right)\end{array}$ & & & \\
\hline 147 & $223557.4+335845.3$ & $5974 \pm 8$ & 0.3 & NSQA \\
\hline 148 & $223557.4+335836.6$ & $5994 \pm 5$ & 0.6 & $\mathrm{~L} 4$ \\
\hline 149 & $223557.4+335734.8$ & $5613 \pm 7$ & 0.5 & NI4 \\
\hline 150 & $223557.0+335734.8$ & $5615 \pm 6$ & 0.4 & NI4 \\
\hline 151 & $223557.0+335735.7$ & $5626 \pm 7$ & 0.4 & NI4 \\
\hline 152 & $223556.8+335736.6$ & $5653 \pm 5$ & 0.7 & NI4 \\
\hline 153 & $223556.6+335737.6$ & $5684 \pm 3$ & 1.4 & NI4 \\
\hline 154 & $223556.5+335817.0$ & $5955 \pm 3$ & 1.2 & $\mathrm{~L} 4$ \\
\hline 155 & $223556.4+335819.9$ & $5911 \pm 4$ & 0.7 & $\mathrm{~L} 4$ \\
\hline 156 & $223556.3+33576.7$ & $5674 \pm 5$ & 0.6 & SDR \\
\hline 157 & $223556.3+335742.9$ & $5717 \pm 4$ & 1.0 & NI4 \\
\hline 158 & $223556.3+335719.4$ & $5699 \pm 8$ & 0.2 & SDR \\
\hline 159 & $223556.2+335744.2$ & $5727 \pm 5$ & 1.4 & NI4 \\
\hline 160 & $223556.1+33577.0$ & $5695 \pm 6$ & 0.3 & SDR \\
\hline $161^{(a)}$ & $223555.9+33597.4$ & $6108 \pm 26$ & 1.9 & L3 \\
\hline $161^{(b)}$ & $223555.9+33597.4$ & $6473 \pm 68$ & 1.9 & L3 \\
\hline 162 & $223556.0+335911.0$ & $6064 \pm 9$ & 0.3 & L3 \\
\hline 163 & $223556.1+335653.1$ & $5625 \pm 10$ & 0.2 & SDR \\
\hline 164 & $223555.6+335737.4$ & $5768 \pm 1$ & 2.8 & NI5 \\
\hline 165 & $223555.5+335743.1$ & $5776 \pm 1$ & 2.5 & NI5 \\
\hline 166 & $223555.4+335744.7$ & $5790 \pm 2$ & 1.4 & NI5 \\
\hline 167 & $223555.3+335733.5$ & $5751 \pm 2$ & 1.7 & NI5 \\
\hline 168 & $223555.3+335710.3$ & $5722 \pm 2$ & 1.1 & SDR \\
\hline 169 & $223555.2+335711.6$ & $5750 \pm 3$ & 0.7 & SDR \\
\hline 170 & $223554.9+33579.7$ & $5705 \pm 3$ & 1.0 & SDR \\
\hline 171 & $223554.3+335715.9$ & $5768 \pm 3$ & 1.3 & SDR \\
\hline 172 & $223554.2+335729.5$ & $5873 \pm 2$ & 1.1 & SDR \\
\hline 173 & $223554.2+335715.3$ & $5786 \pm 2$ & 1.0 & SDR \\
\hline 174 & $223554.1+335716.2$ & $5793 \pm 2$ & 1.4 & SDR \\
\hline 175 & $223553.9+335714.3$ & $5797 \pm 5$ & 0.7 & SDR \\
\hline 176 & $223549.9+335636.5$ & $6247 \pm 6$ & 1.4 & NG \\
\hline
\end{tabular}

